\title{
The maize PIN gene family of auxin transporters
}

\author{
Cristian Forestan, Silvia Farinati and Serena Varotto* \\ Department of Agronomy, Food, Natural Resources, Animal and Environment, University of Padova, Legnaro, Italy
}

\section{Edited by:}

Angus S. Murphy, Purdue University, USA

\section{Reviewed by:}

June M. Kwak, University of Maryland, USA

Christian Luschnig, University of Natural Resources and Life Sciences, Austria

*Correspondence:

Serena Varotto, Department of Agronomy, Food, Natural Resources, Animal and Environment DAFNAE, University of Padova, Viale dell'Università 16, 35020 Legnaro, Padova, Italy. e-mail: serena.varotto@unipd.it
Auxin is a key regulator of plant development and its differential distribution in plant tissues, established by a polar cell to cell transport, can trigger a wide range of developmental processes. A few members of the two families of auxin efflux transport proteins, PINformed (PIN) and P-glycoprotein (ABCB/PGP), have so far been characterized in maize. Nine new Zea mays auxin efflux carriers PIN family members and two maize PIN-like genes have now been identified. Four members of PIN1 (named ZmPIN1a-d) cluster, one gene homologous to AtPIN2 (ZmPIN2), three orthologs of PIN5 (ZmPIN5a-C), one gene paired with AtPIN8 (ZmPIN8), and three monocot-specific PINs (ZmPIN9, ZmPIN10a, and ZmPIN10b) were cloned and the phylogenetic relationships between early-land plants, monocots, and eudicots PIN proteins investigated, including the new maize PIN proteins. Tissue-specific expression patterns of the 12 maize PIN genes, 2 PIN-like genes and ZmABCB1, an ABCB auxin efflux carrier, were analyzed together with protein localization and auxin accumulation patterns in normal conditions and in response to drug applications. ZmPIN gene transcripts have overlapping expression domains in the root apex, during male and female inflorescence differentiation and kernel development. However, some PIN family members have specific tissue localization: ZmPIN1d transcript marks the L1 layer of the shoot apical meristem and inflorescence meristem during the flowering transition and the monocot-specific ZmPIN9 is expressed in the root endodermis and pericycle. The phylogenetic and gene structure analyses together with the expression pattern of the ZmPIN gene family indicate that subfunctionalization of some maize PINs can be associated to the differentiation and development of monocot-specific organs and tissues and might have occurred after the divergence between dicots and monocots.

Keywords: PIN-formed, auxin efflux carriers, kernel development, inflorescences, monocots, polar auxin transport, Zea mays

\section{INTRODUCTION}

Auxin is a key regulator of plant development: its differential distribution in plant tissues, established by a polar transport (polar auxin transport, PAT) in response to internal and external stimuli can trigger a wide range of developmental processes, such as organ initiation at the shoot apex (Reinhardt et al., 2000, 2003; Benkova et al., 2003; Heisler et al., 2005), leaf venation (Scarpella et al., 2006, 2010), apical dominance (Leyser, 2005), tropisms (Blakeslee et al., 2004; Kimura and Kagawa, 2006; Palme et al., 2006), embryo axis formation (Friml et al., 2003; Weijers et al., 2005; De Smet and Jurgens, 2007), root architecture (Blilou et al., 2005; Dubrovsky et al., 2008), and fruit ripening (Ellis et al., 2005). Both metabolic changes and transport of auxin have been demonstrated to play a major role in plant organogenesis and differentiation (Petrasek and Friml, 2009). Auxin is synthesized in many plant tissues throughout several different pathways (Normanly, 2010; Zhao, 2010) and subjected to long-range transport via the vascular system by mass flow. Together with this long-range transport a cell to cell transport is present in the tissues, which covers both long and short distance and is mostly polar according to the chemiostatic theory (reviewed in Zazimalova et al., 2010). Several experimental evidences indicate that the control of auxin transport by carrier proteins is fundamental to patterning processes involving auxin (reviewed in Petrasek and Friml, 2009). Three main types of membrane auxin transporters have been characterized in vascular plants and are responsible for cell to cell auxin movement: the auxin permease 1 (AUX1)/LAX influx carriers (reviewed in Kerr and Bennett, 2007), the ATP-BINDING cassette subfamily B [ABCB; previously known as multidrug resistance (MDR)/Phosphoglycoprotein (PGP)] transporters (reviewed by Geisler and Murphy, 2006; Verrier et al., 2008; Yang and Murphy, 2009) and the PIN-formed (PIN) efflux carriers (reviewed by Tanaka et al., 2006; Vieten et al., 2007; Zazimalova et al., 2007; Krecek et al., 2009; Petrasek and Friml, 2009).

The two families of auxin efflux carriers are characterized by specific features. $\mathrm{ABC}$ is one of the largest and most ubiquitous of the transporter families and its ATP-driven transport is associated with the movements of a wide variety of small molecules, nutrients, and xenobiotics (Verrier et al., 2008). A small group of the $\mathrm{ABCB}$ subclass of $\mathrm{ABC}$ transporters, comprising $\mathrm{ABCB} 1$ and ABCB19 in Arabidopsis thaliana (Blakeslee et al., 2005), function in long-distance auxin transport and localized loading of auxin into the transport system across plant species, while they do not appear to be involved in setting vectorial auxin flows that control organogenesis (reviewed in Titapiwatanakun and Murphy, 2008). The best characterized $A B C B$ auxin transporters in A. thaliana, $\mathrm{ABCB} 1, \mathrm{ABCB} 4$, and $\mathrm{ABCB} 19$, have a stable and non-polar plasma membrane localization in the cell. Particularly, 
Arabidopsis ABCB1/PGP1 has been shown to function in exporting IAA from shoot and root meristematic cells into the long-distance polar auxin stream, and its homologs in maize Brachytic2 (BR2) and sorghum Dwarf3 (DW3) have also been characterized (Multani et al., 2003; Knoller et al., 2010; McLamore et al., 2010). Mutations in BR2 and DW3 genes determine several plant defects, such as altered seedling auxin transport, altered vasculature of the stalk, and reduced stalk height due to shortened lower internodes (Multani et al., 2003; Pilu et al., 2007), confirming the ABCB role in long-range auxin transport. BR2 is also expressed in epidermal and hypodermal tissues at the root apex, where it is involved on shootward auxin transport from the apex to the elongation zone (McLamore et al., 2010). Although some differences exist between Arabidopsis $a b c b 1$ and maize $b r 2$ phenotypes and these differences are likely to reflect differences in developmental patterns, a combination of phylogenetic, phenotypic, and physiological analyses recently showed that $\mathrm{ABCB} 1 / \mathrm{BR} 2$ function is conserved between dicots and monocots (Knoller et al., 2010).

The plant specific PIN family of efflux carriers comprises integral membrane proteins (belonging to the "mem_trans" group of the PFAM database) and has been associated to PAT (Petrasek et al., 2006; Zazimalova et al., 2007; Krecek et al., 2009; Yang and Murphy, 2009). In A. thaliana there are eight PIN genes (AtPIN1-AtPIN8) codifying for proteins that differ in the length of hydrophilic loop in the middle of their polypeptide chain (Krecek et al., 2009; Zazimalova et al., 2010; Peer et al., 2011). The long PIN proteins of Arabidopsis (PIN1-4 and 7) show mostly PMlocalization and their polar localization determines the direction of auxin flux. PIN-mediated PAT regulates many differentiation and developmental processes that have been extensively characterized. Furthermore, long AtPINs do not reside statically in plasma membrane but constitutively cycle between the PM and endosomal compartments and their re-location can be triggered by environmental stimuli (Friml, 2010; Richter et al., 2010). Three PIN proteins of $A$. thaliana, namely PIN5, 6, and 8, have a shorter central hydrophilic domain and both PIN5 and PIN8, in which this domain is particularly short, have been shown to localize in endoplasmic reticulum, suggesting a possible role in regulating intracellular auxin homeostasis (Mravec et al., 2009; Wabnik et al., 2011). The classification of AtPIN6 in long or short PINs is rather more controversial since the hydrophilic loop is only partially reduced while the transmembrane regions show high sequence similarity with long PINs (Krecek et al., 2009; Mravec et al., 2009). In addition to the eight AtPIN proteins, the Arabidopsis genome encodes for seven further PIN-like genes. Phylogenetic analysis revealed that the two families form distinct clusters and the role of these PIN-like proteins has not yet been demonstrated (Paponov et al., 2005).

Many homologous PIN genes were cloned in monocot species and some members of the rice (Oryza sativa; Xu et al., 2005; Wang et al., 2009) and maize (Zea mays; Carraro et al., 2006; Forestan et al., 2010; Forestan and Varotto, in press) families have been characterized. Both specific features and homologies between monocots and Arabidopsis (a eudicot) PIN families have been shown. Monocot-specific features comprise both sequence clustering in phylogenetic analyses and expression pattern at transcript and protein level. In rice, the sequence analysis of the 12
PIN genes present in the genome showed that rice has four PIN1 genes and one OsPIN2, while no OsPIN protein was grouped into the AtPIN3, AtPIN4, AtPIN7 cluster. Four OsPIN genes encode for rice PIN proteins with a short central hydrophilic domain: three OsPIN5 and one OsPIN8. Furthermore, three OsPIN proteins appear monocot-specific: OsPIN9, OsPIN10a, and OsPIN10b. OsPIN9 has a central hydrophilic domain intermediate in length between long and short PINs of Arabidopsis and its expression analysis at transcription level suggests a possible function in adventitious root differentiation. OsPIN10a and OsPIN10b have a long central hydrophilic domain and based on their expression pattern they could be involved in rice tillering (Wang et al., 2009). Until now, three PIN1 genes were identified in maize (ZmPIN1a, $Z m P I N 1 b$, and $Z m P I N 1 c)$ and their transcript and protein localized in different tissues (Carraro et al., 2006; Forestan et al., 2010). Using an antibody raised against the AtPIN1 protein, ZmPIN1 proteins were shown to localize in both a polar and apolar way in the cell plasma membrane in some tissues or to accumulate in the endomembranes inside the cell in a specialized tissue of the endosperm (Forestan and Varotto, 2010; Forestan et al., 2010). Finally, 11 genes belonging to the PIN family have been identified in Sorghum bicolor, confirming that also in this monocot species at least three members of the family are grouped in the AtPIN1 cluster and another three in AtPIN5 cluster (Shen et al., 2010).

In this work we report the identification and characterization of nine new $Z$. mays PIN family members and two maize PIN-like genes. The phylogenetic and gene structure analyses together with the expression pattern of the members of PIN gene family indicate that subfunctionalization of some maize PINs can be associated to the differentiation and development of specific monocot organs and tissues and might have occurred after the divergence between dicots and monocots.

\section{MATERIALS AND METHODS MAIZE AUXIN EFFLUX CARRIERS IDENTIFICATION AND SEQUENCE ANALYSIS}

The B73 maize genome sequence has recently been completed and published (Schnable et al., 2009), and annotated CDS and filtered protein have also become available (release 5b.60). Two genome browsers are available at MaizeGDB ${ }^{1}$ and MaizeSequence ${ }^{2}$ websites. Putative maize auxin efflux carriers were initially identified by Blast searches against the reference genome using $A$. thaliana and $O$. sativa PIN transcript and protein sequences as queries (see Table S1 in Supplementary Material, for accession numbers of all the sequences used in this study). Following this approach, we retrieved the sequences of nine new maize PIN-related genes with their relative annotations (see Table S1 in Supplementary Material, for accessions). Additional Blast searches were performed to identify genomic survey sequences (GSSs), transcripts, and transcript assemblies (TAs) from PlantGDB ${ }^{3}$, from J. Craig Venter Institute ${ }^{4}$, from $\mathrm{NCBI}^{5}$, and from DFCI/TIGR database ${ }^{6}$. A large amount

\footnotetext{
${ }^{1}$ www.maizegdb.org

${ }^{2}$ www.maizesequence.org

${ }^{3}$ http://www.plantgdb.org/cgi-bin/blast/PlantGDBblast

${ }^{4} \mathrm{http}$ ://blast.jcvi.org/tgi_maize/index.cgi

${ }^{5}$ www.ncbi.nlm.nih.gov

${ }^{6} \mathrm{http}: / /$ compbio.dfci.harvard.edu/cgi-bin/tgi/gimain.pl?gudb $=$ maize
} 
of genomic and expressed sequences was retrieved and univocally assigned to each corresponding primary identified sequence by alignment and consensus generation using ClustalX (Thompson et al., 2002), Geneious, and Lasergene DNAStar tools. In addition, GeneSeqer software ${ }^{7}$ was used to assign each TA to its corresponding genomic sequence and indirectly validate the splicing models of genome sequencing derived sequences. Redundant sequences were immediately discarded, while three PlantGDB GSSs without matching sequences in the genome database were further analyzed (AZM5_3988, AZM5_105354, and ZmGSStuc1112-04.463.1). Two of these GSS (AZM5_105354 and AZM5_3988) resulted partial: the first corresponding to the $5^{\prime}$ end and the other to the $3^{\prime}$ end of a putative maize PIN gene and both showing high sequence similarity with a single PIN sequence of $S$. bicolor and, to a lesser extent, with Arabidopsis PIN2. Based on maize/sorghum alignment the two maize GSSs resulted as not overlapping and so the construction of a contig was not possible. However, using primer pairs designed on the putative $5^{\prime}$ UTR and $3^{\prime}$ UTR it was possible to amplify a single genomic and cDNA sequence from B73 and close the gap. This sequence and the third GSS (ZmGSStuc11-12-04.463.1) are not included in the B73 genome databases.

As described for the two partial GSS, several primers (Table S2 in Supplementary Material) were designed on the retrieved full-length sequences and were then used in PCR experiments using B73 inbreed genomic DNA and cDNA as template to directly amplify $Z m P I N$ genes and further validate them. Cloning of the PCR amplification products was performed with the $\mathrm{pCR}^{\circledR} \mathrm{II}-$ TOPO $^{\circledR}$ TA-Cloning kit (Invitrogen) or with the pGEM ${ }^{\circledR}-\mathrm{T}_{\text {Easy }}$ Vector Systems (Promega). E. coli competent cells were transformed by heat-shock and selected on plates containing LB medium, plus ampicillin, and X-Gal (IPTG was added when necessary). Plasmids were then purified using the Plasmid Mini Kit (QiAgen), following the manufacturer's instructions. After sequencing on both strands, cloned sequences were edited, aligned against the reference genome, and translated to putative protein sequences using Geneious and Lasergene DNAStar softwares.

Despite many attempts, the ZmPIN10b full-length cDNA sequences resulted as being un-amplifiable from any of the tissues used in our study, while the genomic sequence was easily amplified and cloned. Curiously no ESTs or TAs corresponding to this gene were found in public databases, suggesting that this gene could be expressed at very low levels or in extremely specific and restricted domains/cell-types (see also the genome-wide atlas of gene expression data reported in Figure S5 in Supplementary Material; Sekhon et al., 2011). A similar situation concerns the $Z m P I N 5 b$ locus. Annotation on genome browser shows a stop codon soon after the junction between the first and second exon, resulting in a truncated protein. Anyway the alignment between $Z m P I N 5 b$ genomic sequence and sorghum ortholog CDS seems to indicate that the CDS predicted in the maize annotation could be wrong: a single base change in the first exon/intron results in the skipping of stop codon and in the "in silico" translation of a

\footnotetext{
${ }^{7}$ http://www.plantgdb.org/cgi-bin/GeneSeqer/PlantGDBgs.cgi
}

functional protein homolog to PIN5 of different species. Unfortunately just the first exon was successfully amplified from cDNA, while several primers designed from the second to the fifth exon sequences did not work in PCR amplifications. As for ZmPIN10b, no EST corresponding to $Z m P I N 5 b$ were retried from databases and no expression has been detected by a genome-wide transcriptome analysis (Figure S5 in Supplementary Material; Sekhon et al., 2011), making it impossible to verify the actual CDS sequence of this gene. Studies described below (phylogenetic and protein profiles analyses) were done using the full-length protein sequence.

\section{PHYLOGENETIC ANALYSIS}

The phylogenetic relationships between newly identified maize PIN proteins and PINs of eudicots and monocots were determined through a robust phylogenetic analysis which also allowed determination of the evolution of the PIN auxin efflux carriers family from early-land plants (mosses) to monocots and eudicots. PIN protein sequences of $A$. thaliana and Medicago truncatula (two herbaceous eudicots), Populus trichocarpa and Vitis vinifera (two arboreal eudicots), Z. mays, O. sativa, S. bicolor, Brachypodium distachyon, and Setaria italica (five monocots), and Physcomitrella patens and Selaginella moellendorffii (a bryophyte and lycophyte, respectively) were retrieved by family blast searches in the Phytozome v7.0 database ${ }^{8}$. All the accessions of the sequences employed in this study are reported in Table S1 in Supplementary Material. Given the large amount of sequences, a subset of "pocket" trees was generated excluding PIN proteins of M. truncatula, V. vinifera, S. bicolor, and S. italica (Figure 1; Figures S2 and S3 in Supplementary Material), while a poster tree with all the sequences is reported in Figure S1 in Supplementary Material.

Evolutionary models of PIN proteins were performed using three different approaches and the results compared. First PIN proteins were aligned with ClustalX 1.81 (Blosum Weight Matrix; Gap Opening Penalty: 5; Gap Extension Penalty: 0.20; Thompson et al., 2002), and a neighbor-joining phylogenetic tree was prepared with Phylip package (Felsenstein, 1989) using 100 bootstraps. In this case the yeast PIN-like protein AEL1 (NP_593365 from Schizosaccharomyces pombe) was included in the analysis as outgroup (Yang and Murphy, 2009; Zazimalova et al., 2010). The phylograms were drawn using Tree-View 1.6.6 $6^{9}$ and reported in Figure 1 and Figure S1 in Supplementary Material. In addition, PIN protein sequences were aligned with MAFFT algorithm (Katoh et al., 2002, 2009) available at the EMBL-EBI website ${ }^{10}$. Phylogeny reported in Figure S3 in Supplementary Material was inferred with the Molecular Evolutionary Genetic Analysis 5 (MEGA5; Tamura et al., 2011) program using the Maximum Likelihood method (10,000 bootstraps, Jones-Taylor-Thornton model). Finally the same alignment was also used to construct a NeighborNet Network (Figure S2 in Supplementary Material) with SplitsTree4 software (Huson and Bryant, 2006).

Based on these phylogenetic analyses, newly identified $Z m P I N$ genes are named according to the cluster of the Arabidopsis and rice PIN family to which they belong.

\footnotetext{
${ }^{8}$ www.phytozome.net/

${ }^{9}$ http://darwin.zoology.gla.ac.uk/wrpage/treeviewx

${ }^{10} \mathrm{http}: / /$ www.ebi.ac.uk/Tools/msa/mafft/
} 


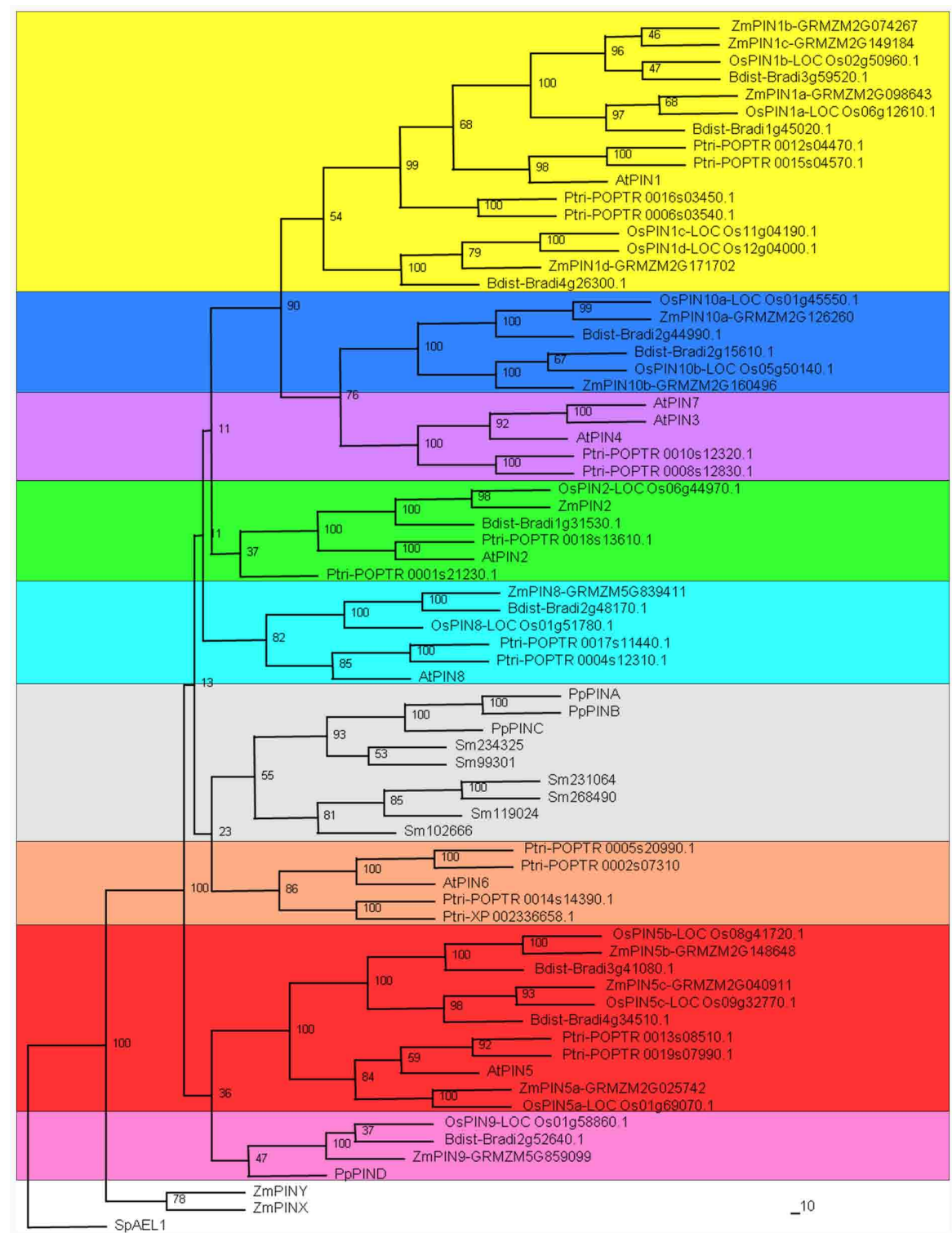

FIGURE 1 | Phylogenetic analysis of PIN auxin efflux carriers from Zea mays, Arabidopsis thaliana, Oryza sativa, Brachypodium distachyon, Populus trichocarpa, Physcomitrella patens, and Selaginella

moellendorffii. The phylogenetic analysis between newly identified maize PIN proteins and PINs of eudicots and monocots, summarizes the evolutionary relationships among the 70 members of the PIN protein family. PIN protein sequences of $Z$. mays $(Z m), A$. thaliana $(A t), P$. trichocarpa (Ptri), O. sativa (Os), B. distachyon (Bdist), P. patens (Pp), and $S$. moellendorffii $(\mathrm{Sm})$ were retrieved by family blast searches in the
Phytozome v7.0 database (www.phytozome.net/). Accession numbers are reported in Table S1 in Supplementary Material. PIN proteins were aligned with ClustalX 1.81 (Blosum Weight Matrix; Gap Opening Penalty: 5; Gap Extension Penalty: 0.20), and a neighbor-joining phylogenetic tree was prepared with Phylip package using 100 bootstraps. The yeast PIN-like protein AEL1 (NP_593365 from Schizosaccharomyces pombe) was included in the analysis as outgroup. Newly identified ZmPIN proteins are named according to the cluster of the Arabidopsis and rice PIN family to which they belong.

\section{GENE STRUCTURES, MAP POSITIONS, DUPLICATION ANALYSIS, AND PROTEIN PROFILE ANALYSIS}

Exon/intron structures of maize PIN genes were obtained from the MaizeGDB Genome browser ${ }^{11}$, except for $Z m P I N 2$ and $Z m P$ $I N X$ that are not present in the genome database and for which exon/intron profiles have been drawn with GSDS, Gene Structure

\footnotetext{
${ }^{11}$ http://gbrowse.maizegdb.org/cgi-bin/gbrowse/maize_v2/
}

Display Server (Guo et al., 2007) ${ }^{12}$ based on the alignment between the previously amplified and sequenced genomic and cDNA sequences.

ZmPIN map positions were identified by searching the www.maizesequence.org database and are manually depicted in Figure 2 and Figure S4 in Supplementary Material; the exact

\footnotetext{
${ }^{12}$ http://gsds.cbi.pku.edu.cn/index.php
} 


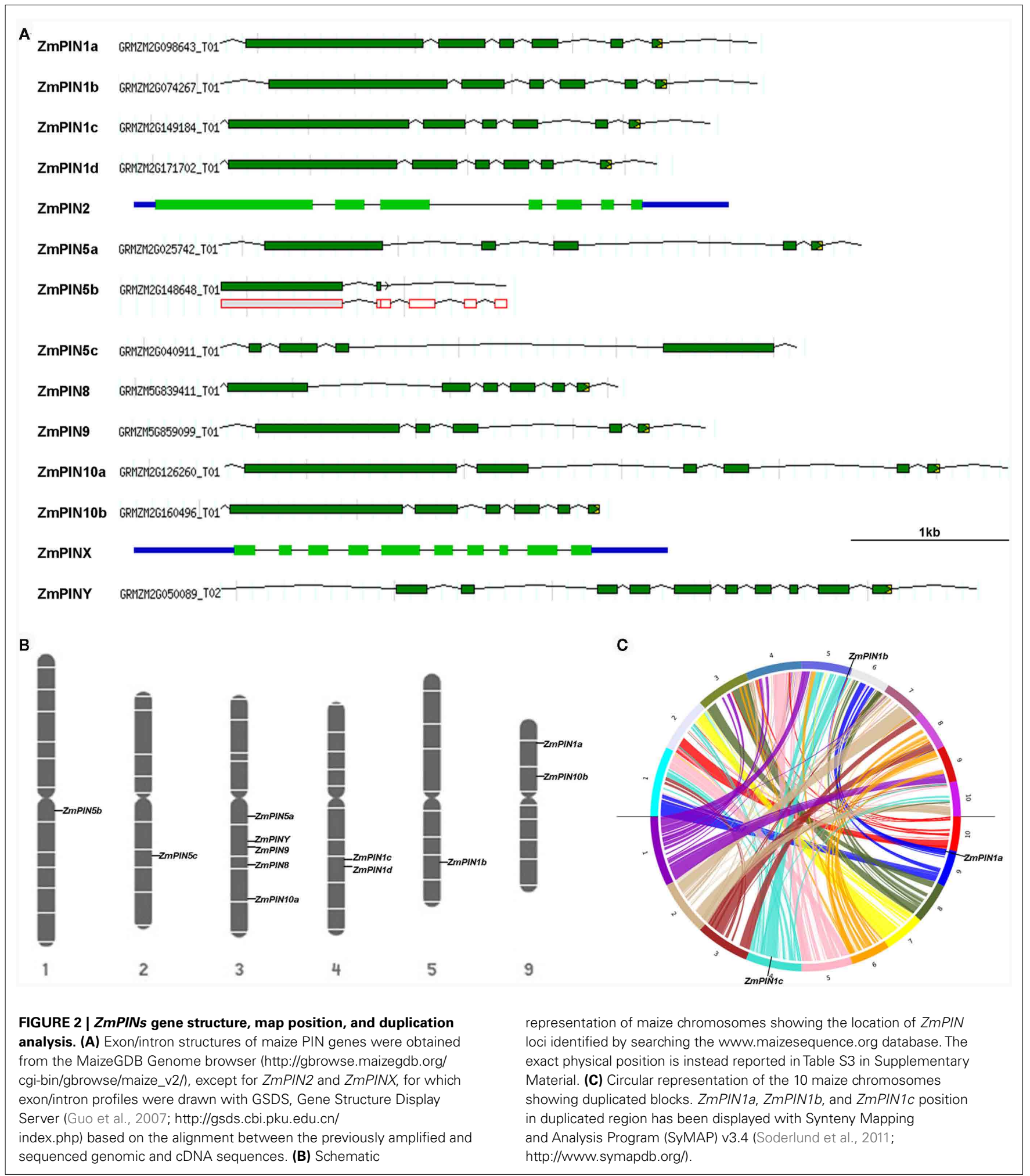

physical position is instead reported in Table S3 in Supplementary Material.

Duplication analysis of maize PIN genes and synteny between maize and rice chromosomes were identified and displayed by

Synteny Mapping and Analysis Program (SyMAP) v3.4 (Soderlund et al., 2011) $)^{13}$

${ }^{13} \mathrm{http}: / /$ www.symapdb.org/. 
Finally, ZmPIN proteins transmembrane helices were predicted using TMHMM2 (Krogh et al., 2001) ${ }^{14}$ : the red peaks in Figure 3 show the predicted transmembrane domains of proteins.

\section{PLANT MATERIAL}

Zea mays B73 line was used for DNA and RNA extraction, for expression analysis, immunolocalization, and in situ hybridization. Plants were grown in the field or greenhouse, with supplemental lighting when necessary during winter/spring. Kernels were harvested and analyzed at different days after pollination (DAP) after manual self-pollination of plants in the field trials. Roots were collected 2-3 days after kernel germination in rolled adsorbent paper towels soaked with water in a growth chamber at $26^{\circ} \mathrm{C}$ with a photoperiod of $16 \mathrm{~h}$ light and $8 \mathrm{~h}$ dark as described in Woll et al., 2005. Kernels were previously surface sterilized with $70 \%$ ethanol for $10 \mathrm{~min}$ and then rinsed three times with water.

The maize mutant line br2 (Multani et al., 2003; Pilu et al., 2007) was kindly supplied by Dr. Roberto Pilu (Università di Milano) after introgression for six generations in B73 background. Homozygous br2 mutant plants were grown as previously described. Furthermore, the reporter lines pZmPIN1a::ZmPIN1a:YFP and DR5::mRFP (Gallavotti et al., $2008)^{15}$, kindly provided by Prof. Dave Jackson, were introgressed for three additional generations in B73 keeping the transgene in a hemizygous state.

\section{NUCLEIC ACID EXTRACTION}

Genomic DNA was extracted from maize leaves with the DNeasy Plant Mini Kit (QiAgen) according to the manufacturer's instructions. Total RNA was extracted from maize tissues (seedlings, roots, leaves, tassels, ears, and kernels) using to the RNeasy Plant Mini Kit (QiAgen) and subjected to on-column DNase treatment (QiAgen). Before retrotranscription, total RNA was quantified by spectrophotometer analysis.

\section{EXPRESSION ANALYSES BY RT-PCR, REAL-TIME PCR, AND IN SITU HYBRIDIZATION ASSAYS}

For the semi-quantitative RT-PCR expression analysis, ZmPIN and $Z m A B C B 1 / B R 2$ gene transcriptions were investigated in vegetative and reproductive tissues and kernels. Vegetative tissues included 3 days-old seedlings, young leaves, the apex (from 0 to $1.5 \mathrm{~cm}$ ) and the elongation/mature zone (from 1.5 to $3 \mathrm{~cm}$ ) of primary roots, and the seventh and fifth node, while for the reproductive tissues ear and tassel at the stage of 1 and $3 \mathrm{~cm}$ were considered. Kernels collected at different DAP $(0,3,5,6,7,8$, $9,10,12)$ were also taken into account for the $Z m P I N$ expression analysis.

For the detailed quantitative PINs expression analysis in B73 line and br2 mutant, young roots were collected from seedlings vertically grown in a growth chamber for 3 days as previously described. These roots were divided into specific segments, which were labeled from A to E indicating the segment from 0 to $0.2 \mathrm{~cm}$ (A), from 0.2 to $0.5 \mathrm{~cm}(\mathrm{~B})$, from 0.5 to $1 \mathrm{~cm}(\mathrm{C})$, from 1 to $1.5 \mathrm{~cm}$

\footnotetext{
${ }^{14} \mathrm{http}: / /$ www.cbs.dtu.dk/services/TMHMM-2.0/

${ }^{15} \mathrm{http}: / /$ maize.jcvi.org/cgi-bin/maize/cellgenomics/geneDB_list.pl
}

(D), and from 1.5 to $2 \mathrm{~cm}$ (E; Figure 6A). In these root segments the expression of $Z m P I N$ genes and $Z m A B C B 1 / B R 2$ was investigated by Real-Time PCR.

For both expression analyses, cDNA synthesis was performed with the SuperScript III reverse transcriptase kit (Invitrogen), according to the manufacturer's instructions. One microgram of total RNA was used as a template together with $1 \mu$ l oligo (dT) $12-18(0.5 \mu \mathrm{g} / \mu \mathrm{l}$ - Invitrogen $)$. In the semi-quantitative RT-PCR expression analysis, each cDNA was then diluted $1: 10$ and $1 \mu \mathrm{l}$ was used for PCR amplification in a volume of $25 \mu \mathrm{l}$. The constitutively expressed GAPC2 gene was used as housekeeping internal control of the cDNA/RNA quantity. Expression analyses were performed with at least two primer combinations for each gene. Primer sequences are reported in Table S2 in Supplementary Material. In order to obtain semi-quantitative results, the number of cycles of the PCR was adjusted for each gene to obtain barely visible bands in agarose gels (see Table S2 in Supplementary Material for the number of amplification cycles for each gene). Aliquots of the PCR were loaded on 1.2\% agarose gels and stained with Sybr-Safe (Invitrogen). For all PINs the different primer combinations tested gave comparable amplification results. $Z m A B C B 1 / B R 2$ amplification products (see Pilu et al., 2007 for primers used in RT-PCR amplification) were checked on $2 \%$ agarose gel. Images were captured with Gel Logic 100 Imaging System and Molecular Imaging software (Kodak).

Quantitative Real-Time PCR expression analysis was performed using an ABI 7500 Real-Time PCR System (Applied Biosystems) and the POWER SYBR ${ }^{\circledR}$ GREEN PCR Master Mix (Applied Biosystems), following the manufacturer's guidelines. Real-time conditions were: $2 \mathrm{~min}$ at $50^{\circ} \mathrm{C}, 10 \mathrm{~min}$ at $95^{\circ} \mathrm{C}, 40$ cycles of: $15 \mathrm{~s}$ at $95^{\circ} \mathrm{C}$ and $1 \mathrm{~min}$ at $60^{\circ} \mathrm{C}$. For each reaction, we observed product melting curves by heating from 60 to $95^{\circ} \mathrm{C}$ at $0.2^{\circ} \mathrm{C} / \mathrm{s}$. For all transcripts, this procedure allowed identification of a single product, which we confirmed by analysis on $2 \%$ agarose gel. Two different biological samples, obtained by different RNA preparations from separate root pools, were processed; three replicates were carried out for each primer combination and for each biological sample. The absolute quantification was carried out by the generation of standard curves for each gene. Expression levels of each auxin transporter gene were normalized to GAPC2 transcript quantities. Primer sequences are reported in Table S2 in Supplementary Material.

In situ hybridization experiments were conducted as described by Varotto et al., 2003. Plant material (kernels, vegetative apexes, roots, and inflorescences) was fixed in $4 \%$ paraformaldehyde (Sigma) in $0.1 \mathrm{M}$ phosphate buffer $(\mathrm{pH} 7.2)$ for $16 \mathrm{~h}$ at $4^{\circ} \mathrm{C}$, then dehydrated by ethanol series and embedded in Paraplast Plus (Sigma). Sections $(7-10 \mu \mathrm{m})$ were cut using a Leica RM 2135 microtome (Leica, Germany) and collected on xylanecoated SuperFrost Plus slides (Menzel-Glazer). Slides were then deparaffinized and treated with $10 \mu \mathrm{g} / \mathrm{ml}$ proteinase $\mathrm{K}$.

In vitro transcription of the DIG-UTP (Roche) labeled RNA sense and antisense probes was obtained using T7 and SP6 polymerases. More information on primer sequences used to amplify the probes for each PIN gene can be found in Table S2 in Supplementary Material. The hybridization was performed in a $50 \%$ formamide buffer at $48^{\circ} \mathrm{C}$ overnight. DIG detection and 

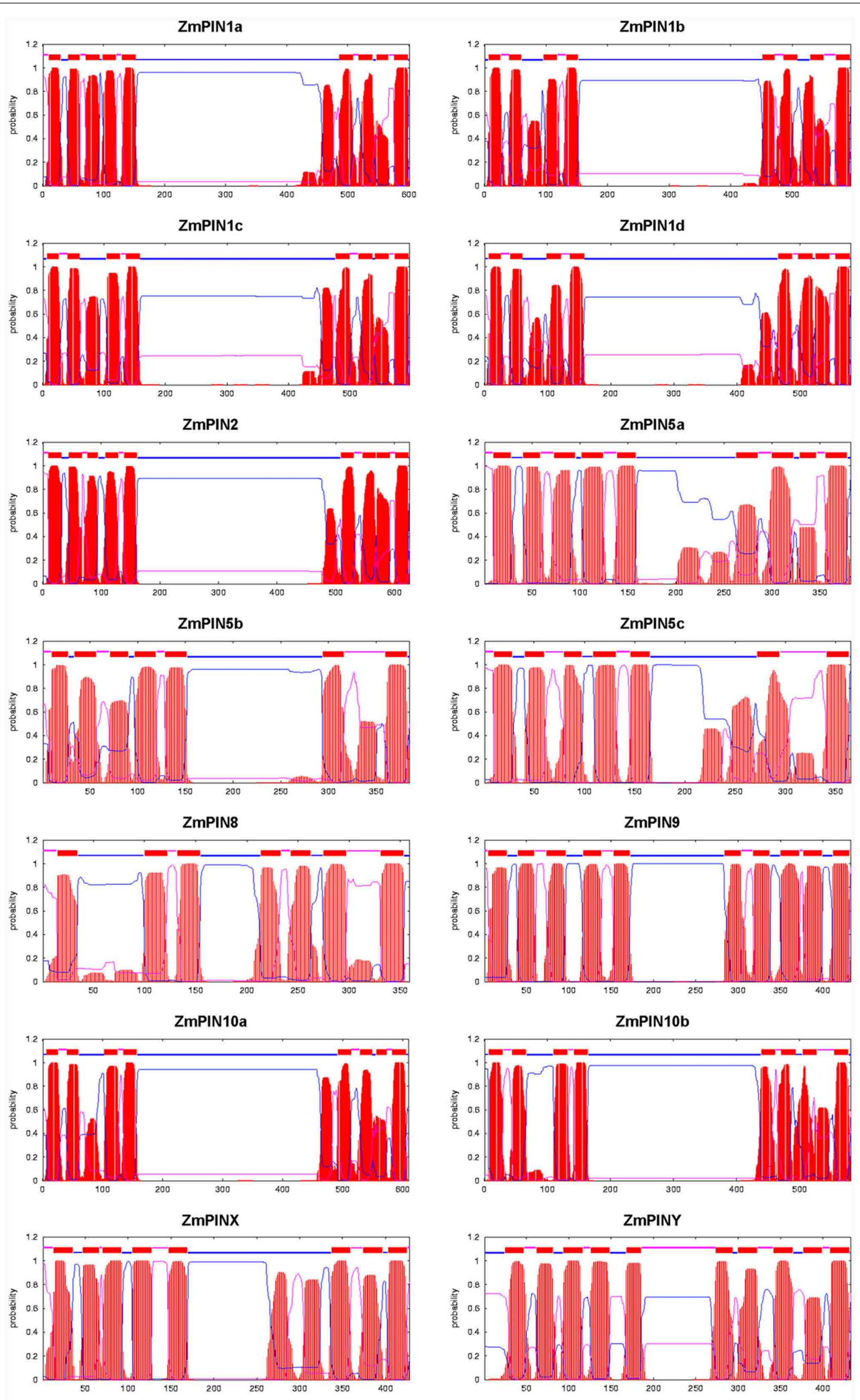

transmembrane

inside $-\quad$ outside -

FIGURE 3 |The predicted protein profiles of the maize PIN transporters. The transmembrane helices of PIN proteins were predicted using TMHMM2

(Krogh et al., 2001; http://www.cbs.dtu.dk/services/TMHMM-2.0/): the red peaks show the predicted transmembrane domains of proteins. 
signal visualization were done using Anti-Digoxigenin-AP antibody (Roche) and NBT plus BCIP (Roche), following the manufacturer's instructions. Slides were air-dried and mounted with DPX mounting medium (Fluka Biochemika). Negative controls obtained with sense riboprobes are reported in Figures S6A-D in Supplementary Material.

\section{MOLECULAR ANALYSES UNDER NPA AND NAA TREATMENTS}

Zea mays B73 line and br2 mutant seeds were surface sterilized with $70 \%$ ethanol for $10 \mathrm{~min}$ and then rinsed three times with sterile water. Kernels were placed on soaked 3MM paper in horizontal position for 1 day. After imbibition, the seeds were transferred in rolled adsorbent paper towels in vertical position for successive treatments. Seedlings were treated with $50 \mu \mathrm{M}$ NPA (1-naphthylphthalamic acid, a PAT inhibitor), $50 \mu \mathrm{M}$ NAA (2naphthalen-1-ylacetic acid, a synthetic auxin), and water as control growth condition for 2 days. Roots were collected and divided in two specific segments indicated with A (from 0 to $0.2 \mathrm{~cm}$ ) and $\mathrm{B}$ (from 0.2 to $0.5 \mathrm{~cm}$ ).

The expression profiles of $Z m P I N$ genes and $Z m A B C B 1 / B R 2$ in untreated, NPA- and NAA-treated roots were investigated by Real-Time PCR using the standard curve method, as reported above.

The reporter lines pZmPIN1a::ZmPIN1a:YFP and DR5::mRFP were treated with NPA and NAA at the same concentrations and observed at the confocal microscope.

\section{IMMUNOLOCALIZATION ASSAYS}

PIN1 immunostaining was performed on wax-embedded (9:1 PEG400 distearate:1-hexadecanol 99\%, Sigma) roots collected on 2-3 days-old plants vertically grown on rolled paper. Roots were fixed in 4\% paraformaldehyde in PBS $1 \times$ solution for $1 \mathrm{~h}$ under vacuum conditions at room temperature, embedded, and cut (10$12 \mu \mathrm{m}$ ) with a Leica RM 2135 microtome (Leica, Germany). After dewaxing sections were blocked for $2 \mathrm{~h}$ in $1 \%$ BSA in $1 \times$ PBS buffer and stained overnight with the anti-AtPIN1 antibody (aP20 sc-27163, Santa Cruz Biotechnology) at a 1:250 dilution in PBS $1 \times$ and $1 \%$ BSA. The next day, the sections were washed twice for $10 \mathrm{~min}$ in PBS $1 \times$ and stained for $1 \mathrm{~h}$ with the secondary antibody (anti-goat IgG conjugated with Alexa568, Molecular Probes) diluted 1:400 in PBS 1×. Sections were washed twice for 10 min with PBS $1 \times$ and mounted with VECTASHIELD ${ }^{\circledR}$ with DAPI Mounting Medium (Vector Laboratories, USA). For the negative controls of immunostaining experiments hybridization was performed with the secondary antibody only.

A monoclonal anti-IAA antibody (Biofords - Agdia PMD09346/0096) was used for IAA immunolocalization in maize roots. Tissues were prefixed in $3 \%(\mathrm{w} / \mathrm{v}) \mathrm{N}$-Ethyl- $N^{\prime}$ (3-dimethylaminopropyl)carbodiimide hydrochloride (EDAC, Sigma) $0.1 \mathrm{M}$ in PBS $1 \times$, post-fixed in $4 \%$ paraformaldehyde in the same buffer and embedded in Paraplast Plus (Sigma). Sections $(7-10 \mu \mathrm{m})$ were cut using a Leica RM 2135 microtome (Leica, Germany), collected on polysine slides (Menzel-Glazer) and processed as previously reported by Avsian-Kretchmer et al., 2002. The anti-IAA primary antibody was used at a concentration of $0.05 \mathrm{mg} / \mathrm{ml}$. The secondary antibody, an anti-mouse IgG-AP
(Santa Cruz Biotechnology), was used at a 1:250 dilution. Signal visualization was done using SIGMAFAST ${ }^{\mathrm{TM}}$ Fast Red Tablets (Sigma) and when purple was observed, the colorimetric reaction was stopped in water. Negative controls of IAA immunostaining experiments were performed on samples no pre-fixed with EDAC or hybridizing slides with the IgG-AP secondary antibody only and are reported in Figures S6E-G in Supplementary Material.

\section{MICROSCOPY AND REPORTER LINES OBSERVATIONS}

pZmPIN1a::ZmPIN1a:YFP and DR5::mRFP reporter lines roots were hand sectioned, mounted with $50 \%$ glycerol in water and observed with a Leica TCS SP2 laser confocal microscope (Leica Microsystems, Heidelberg, Germany) using the follow settings: (i) ZmPIN1a-YFP excitation at $514 \mathrm{~nm}$ and signal collection between 530 and $580 \mathrm{~nm}$; (ii) DR5 excitation at $543 \mathrm{~nm}$ and signal collection between 580 and $640 \mathrm{~nm}$. Images were coded red for RFP and yellow for the YPF reporter line.

Immunolocalization and in situ hybridization images were taken with a Leica DM4000B Digital microscope, equipped with a Leica DC300F Camera, and Leica Image Manager 50 software (Leica Microsystems, England). Images were coded red for Alexa568 and blue for DAPI.

\section{RESULTS}

THE MAIZE PIN FAMILY OF AUXIN EFFLUX CARRIERS: IDENTIFICATION, PHYLOGENETIC ANALYSIS, GENE STRUCTURES, AND PROTEIN PROFILES

Members of the maize PIN family were initially identified by a comprehensive Blast search in public databases using A. thaliana and $O$. sativa PIN transcript and protein sequences as queries. MaizeGDB and MaizeSequence were used as primary databases to obtain the genomic sequences coming from the B73 Maize Genome Sequencing project, release 5b.60, and their relative annotations, while GSSs from PlantGDB and from J. Craig Venter Institute, transcript sequences from NCBI and TAs from DFCI/TIGR were used to initially confirm and integrate the previously identified sequences and splicing models. In addition to the three ZmPIN1 genes previously characterized (Carraro et al., 2006; Forestan et al., 2010), nine full-length sequences were found in the genomic release $5 \mathrm{~b} .60$ and a tenth sequence was identified in a GSS from PlantGDB. Furthermore, two partial, not overlapping, GSSs were assembled together using a sorghum PIN sequence to fill the gap, for a total of 11 new putative auxin efflux carrier genes. A preliminary in silico characterization was performed to identify putative ORFs and amino acid sequences. All the fulllength sequences were later amplified from genomic DNA and cDNA (except ZmPIN5b and ZmPIN10b for which the amplification of the cDNA full-length proved unsuccessful), to validate the sequences of the 11 maize PINs that were named according to the cluster of Arabidopsis and rice PIN family to which they belonged.

The phylogenetic relationships between early-land plants, monocots and eudicots PIN proteins were investigated including the new maize PIN proteins in a phylogenetic analysis (Figure 1; Figures S2 and S3 in Supplementary Material), together with PIN proteins of A. thaliana, $P$. trichocarpa, O. sativa, B. distachyon, $P$. patens, and $S$. moellendorffi. A wider evolutionary study was performed by also including PIN proteins of M. truncatula, $V$. vinifera, 
S. bicolor, and S. italica (Figure S1 in Supplementary Material). PIN protein sequences of different species were retrieved from Phytozome v7.0 and analyzed with different sequence alignment and phylogenetic analysis tools, to develop a robust evolutionary model of transporters. Auxin efflux carrier family members were aligned with ClustalX 1.81 (Blosum Weight Matrix; Gap Opening Penalty: 5; Gap Extension Penalty: 0.20; Thompson et al., 2002) and a neighbor-joining phylogenetic tree was prepared with the Phylip package (Felsenstein, 1989) using 100 bootstraps (Figure 1; Figure S1 in Supplementary Material). In addition, PIN proteins were aligned with MAFFT algorithm (Katoh et al., 2002, 2009) and phylogeny inferred using MEGA5 program (Maximum Likelihood method; 10,000 bootstraps; Jones-Taylor-Thornton model; Tamura et al., 2011; Figure S3 in Supplementary Material) or constructing a NeighborNet Network with SplitsTree4 software (Huson and Bryant, 2006; Figure S2 in Supplementary Material). The three different approaches showed comparable results on the evolutionary history of auxin efflux carrier proteins in earlyland plants, monocots and dicots, also suggesting the evolutionary relationships between the 11 newly identified maize proteins, Arabidopsis, and rice PIN proteins (Figure 1; Figures S1-S3 in Supplementary Material). Indeed, the four different phylogenetic trees show the same clustering of newly identified maize PIN proteins with Arabidopsis and rice orthologs, while differences in few nodes with low support can be observed for $V$. vinifera and $P$. trichocarpa proteins (Figure 1; Figures S1-S3 in Supplementary Material).

In addition to the previously characterized ZmPIN1a, $Z m P I N 1 b$, and ZmPIN1c, a fourth gene phylogenetically close to AtPIN1 was identified. We named this gene, which clusterizes together with rice OsPIN1c and OsPIN1d genes, ZmPIN1d. The PlantGDB database search and the phylogenetic analysis also allowed identification of a putative ZmPIN2 gene; indeed the two available partial GSSs codify for a protein closely related to AtPIN2 and OsPIN2.

The gene structure analysis showed that also the fourth ZmPIN1 gene, $Z m P I N 1 d$, has six exons, whose distribution is similar to that of AtPIN1 and the four OsPIN1 genes (Figure 2A). Like the Arabidopsis and rice orthologs, ZmPIN2 gene presents a complex exon/intron structure, with seven exons and six introns. We also identified four genes that encode for proteins lacking the central hydrophilic domain that normally characterizes long PIN proteins. This peculiar hydropathic profile is typical of the Arabidopsis PIN5 and PIN8 proteins and our phylogenetic analysis confirmed that three of the maize proteins clusterize with Arabidopsis and rice PIN5 (ZmPIN5a, ZmPIN5b, and ZmPIN5c), while the remaining one represents the maize PIN8 ortholog ( $Z m P I N 8)$. So, as recently reported in rice, three duplicated PIN5 genes and a PIN8 gene are also present in the maize genome. ZmPIN5a and ZmPIN5b have four introns, while $Z m P I N 5 c$ has three with the first of about $2 \mathrm{~kb}$ in length (Figure 2A). As described in the Section "Material and Methods," genome annotation of PIN5b gene showed the presence of a stop codon soon after the first splicing site, resulting in a truncated protein (Figure 2A). Anyway, the alignment with the sorghum ortholog CDS showed that the splicing model annotated could be wrong and a shift of a single base pair resulted in a full-length PIN5 protein. Unfortunately RT-PCR using several primer combinations proved unsuccessful in the amplification of the complete CDS. Moreover no ESTs or TAs corresponding to $Z m P I N 5 b$ were found on public databases and a recent GenomeWide Expression Atlas revealed that ZmPIN5b is not expressed in the 60 distinct analyzed tissues representing 11 major organ systems of inbred line B73 (Figure S5 in Supplementary Material; see text footnote 1; Sekhon et al., 2011). ZmPIN8 has a very similar exon/intron structure to that of OsPIN8 (Figure 2A).

Finally, we identified three proteins that do not clusterize with any Arabidopsis PIN proteins, confirming, as suggested for rice, the existence of monocot-specific PIN genes. Based on the phylogenetic analysis, these maize genes were named $Z m P I N 9, Z m P I N 10 a$, and ZmPIN10b. These monocot-specific PIN genes have a conserved structure when compared to their rice counterparts. Phylogenetic analyses also revealed that ZmPINX and ZmPINY proteins clusterize together with the Arabidopsis PIN-like proteins (Figure S1 in Supplementary Material).

Chromosome map positions of maize PINs have been identified by genome browser searches and are reported in Figure 2B and Figure S4 in Supplementary Material. Duplication analysis revealed that $Z m P I N 1 a, Z m P I N 1 b$, and $Z m P I N 1 c$ are located in duplicated blocks on chromosomes 9, 5, and 6, respectively (Figure 2C; Figure S4 in Supplementary Material). Interestingly, both maize and rice PIN5a, PIN8, PIN9, and PIN10a map on highly syntenic regions of chromosomes 3 and 1, respectively (Figure S4 in Supplementary Material, Wang et al., 2009).

The predicted transmembrane architecture of the ZmPIN proteins showed the typical structure with a conserved N-terminal region of transmembrane motifs, a variable middle domain representing the cytoplasmic domain of transmembrane and a Cterminal domain of transmembrane segments (Figure 3). Interestingly, ZmPIN9 putative protein presents a hydropathic profile in between that of long PINs (AtPIN1 and AtPIN2, which are plasma membrane localized) and that of short PINs (the ER-localized AtPIN5 and AtPIN8).

\section{EXPRESSION ANALYSIS OF MAIZE AUXIN TRANSPORTERS}

The tissue-specific expression pattern of $12 \mathrm{ZmPIN}$ genes (ZmPIN1a, ZmPIN1b, ZmPIN1c, ZmPIN1d, ZmPIN2, ZmPIN5a, ZmPIN5b, ZmPIN5c, ZmPIN8, ZmPIN9, ZmPIN10a, and ZmPIN10b), two ZmPIN-like genes (ZmPINX and ZmPINY) and $B R 2$, a maize $M D R / P G P / A B C B$ efflux transporter were analyzed by semi-quantitative RT-PCR and the results are reported in Figure 4. These results are in agreement with those obtained by the genomewide transcriptome analysis previously mentioned and reported in Figure S5 in Supplementary Material (Sekhon et al., 2011).

ZmPIN1a, ZmPIN1b, and ZmPIN1c expression analysis confirmed that these genes are ubiquitously expressed but differentially modulated in maize vegetative and reproductive tissues and during kernel development (see also Carraro et al., 2006; Forestan et al., 2010). A different expression pattern was observed for ZmPIN1d that is specifically expressed in tassels, ears and in the fifth node of adult plants and not detectable in caryopses after pollination. ZmPIN2 is expressed in root tip, male and female inflorescences and its expression is modulated during the early stages of kernel development. ZmPIN5a is mainly expressed in the elongation/mature zone of the primary root, in nodes and in the young seed till 10 DAP. ZmPIN5b is up-regulated in the 


\section{Vegetative \& Reproductive Tissues}

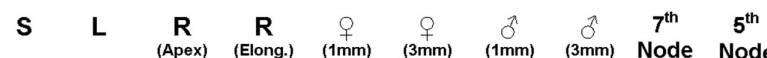
W

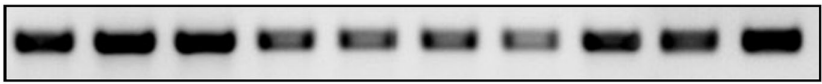

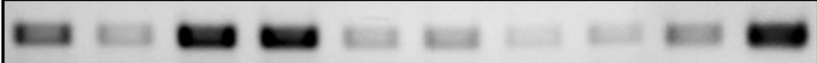
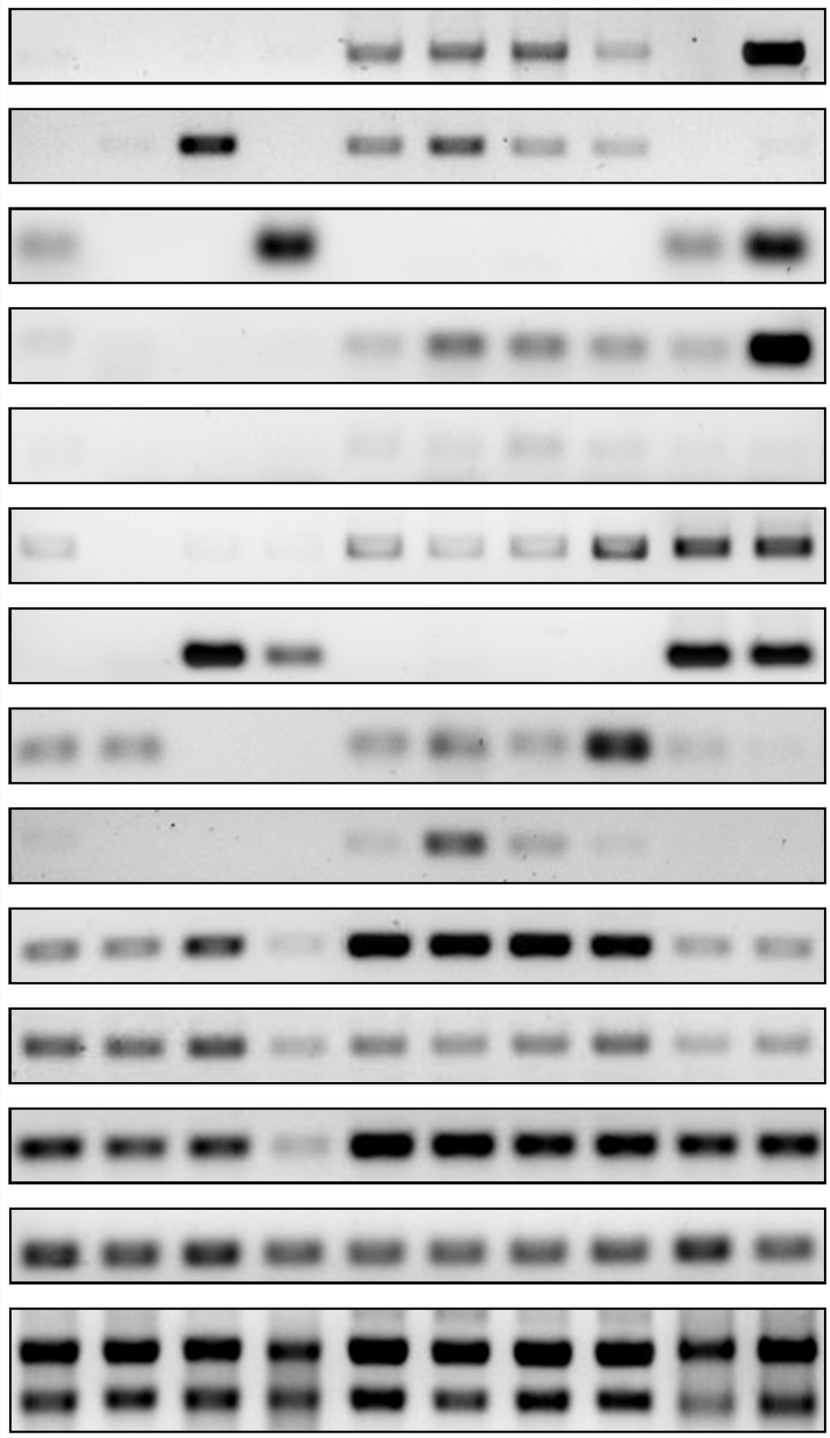

FIGURE 4 | Expression analysis of maize auxin transporters.

Semi-quantitative RT-PCR analysis of $12 \mathrm{ZmPIN}$ genes, two maize PIN-like genes and $B R 2$, a maize MDR/PGP/ABCB efflux transporter in vegetative and reproductive tissues and in kernels from 0 to 12 days after pollination (DAP). The constitutively expressed GAPC2 gene was used as housekeeping internal control of the RNA/cDNA quantity. To get semi-quantitative results, the number of cycles of the PCR was adjusted for each gene to obtain barely visible bands in agarose gels (see Table S2 in Supplementary Material for the

\section{Kernels}

Days After Pollination

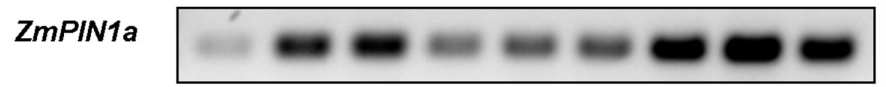

ZmPIN1b

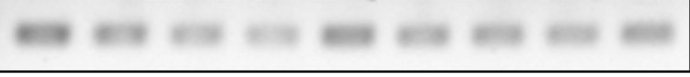

ZmPIN1c

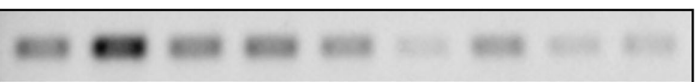

ZmPIN1d

ZmPIN2
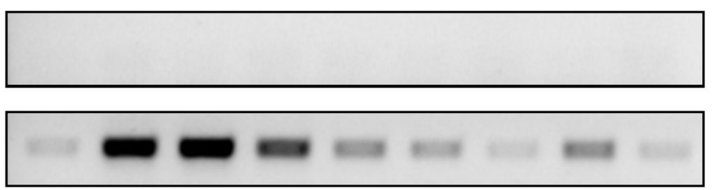

ZmPIN5a

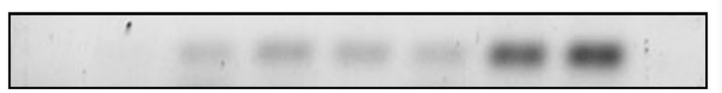

ZmPIN5b

ZmPIN5c
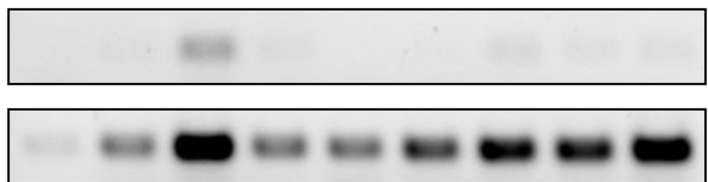

ZmPIN8

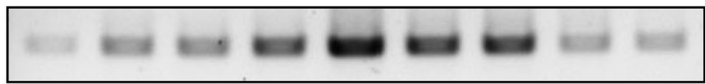

ZmPIN9

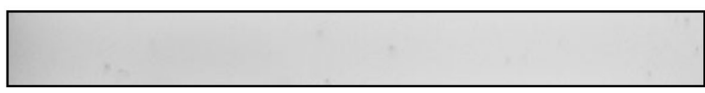

ZmPIN10a

ZmPIN10b

ZmPINX

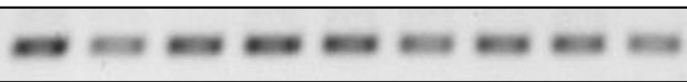

ZmPINY

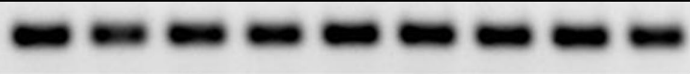

$Z m A B C B 1$ $B R 2$

GAPC2
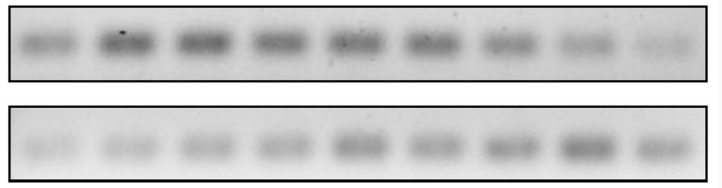

RNA

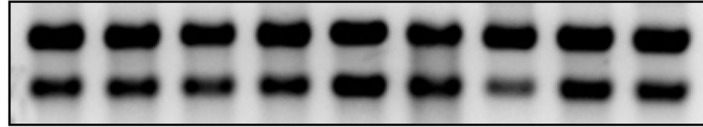

number of amplification cycles for each gene). ZmPIN1a, ZmPIN1b, and ZmPIN1C are expressed ubiquitously and differentially up-regulated in the tissues. ZmPIN1d is expressed in tassels, ears, and in the 5th node of adult plants. ZmPIN2 is expressed in root apex (R), in male and female inflorescences and during maize kernel development. ZmPIN5a is expressed in seedlings (S), in the root elongation/mature zone, in nodes and in the young seed till 10 DAP. ZmPIN5b is expressed in male and female inflorescences,

(Continued) 


\section{FIGURE 4 | Continued}

in nodes and at a low level during kernel development. ZmPIN5C is highly expressed during kernel development. ZmPIN8 is expressed in male and female inflorescences during kernel development. ZmPIN9 is expressed in roots and nodes. ZmPIN10a is expressed in seedlings and leaf (L), in male and female inflorescences and at low level during kernel development. ZmPIN10b is expressed in ears and tassels. ZmPINX and ZmPINY are both expressed ubiquitously and differentially up-regulated in the tissues. BR2 shows a constitutive expression but it appeared down-regulated in the root elongation zone in comparison with root apexes. fifth node, and has a weak expression in male and female inflorescences and during kernel development (note that the amplified fragment corresponds to the first exon while no amplifications were obtained with downstream located primers). ZmPIN5c is highly expressed during kernel development from 3 to 12 DAP. ZmPIN8 is expressed in all the tissues analyzed with the exception of the root and is detectable during kernel development, where it appeared up-regulated between 6 and 9 DAP. Of the monocot-specific genes, ZmPIN9 is exclusively expressed in roots and nodes while $Z m P I N 10 a$ is expressed during male and female inflorescence development and at very low level during kernel development. Finally a very weak expression of $Z m P I N 10 b$ is detectable in young ears and tassels.

The expression analyses of the two maize PIN-like genes showed that they are ubiquitously expressed and differentially up-regulated in all the analyzed tissues. In detail, ZmPINX is upregulated in root apex and male and female inflorescences, while $Z m P I N Y$ is highly expressed during kernel development.

Finally, BR2 transcript is detectable in all the tested tissues with a lower expression level in the root elongation zone compared to other vegetative and reproductive tissues. Moreover, the BR2 transcript amount is detectable at a very low level during tested kernel development stages.

\section{LOCALIZATION OF PIN TRANSPORTERS AND AUXIN MAXIMUM IN MAIZE PRIMARY ROOTS}

The longitudinal structure of the maize root includes the root cap at the terminal end, the root apical meristem, and the elongation and root hair zones. In cross section, the root is organized in a series of concentric cell layers: the outermost epidermic layer and exodermis, the pluristratified cortex, the endodermis, and the pericycle that surrounds the central vascular cylinder (see Hochholdinger et al., 2004 for a review on root development in maize). Six $Z m P I N$ genes are expressed in maize root: indeed, quantitative RT-PCR data showed that ZmPIN1a, ZmPIN1b, and ZmPIN1c and the monocot-specific ZmPIN9 are expressed both in root apical and distal zones (Figure 4). In addition, ZmPIN2 appeared up-regulated in root apex, while $Z m P I N 5 a$ is up-regulated in the root elongation/mature zone (Figure 4). The expression pattern of $Z m P I N$ genes expressed in the root apex was determined at tissue level using in situ hybridization (Figures 5A-O). In root apex longitudinal sections, comprising the root cap and meristematic area, a $Z m P I N 1$ probe, which recognized the three gene transcripts expressed in the root showed the hybridization signal in the root cap with a clear up-regulation in calyptrogen, in the root meristematic area, in the epidermis and in the differentiating vascular tissues of the central cylinder (data not shown). Specific probes for $Z m P I N 1 a, Z m P I N 1 b$, and $Z m P I N 1 c$ were also transcribed in vitro DIG-labeled and hybridized on root apex longitudinal sections. The results showed that ZmPIN1a is the only
PIN1 transcript localized in the calyptrogen and in the meristem region of the root (Figure 5A), while ZmPIN1b is mainly expressed in epidermis, root cap, and vasculature (Figures 5B-D). Finally a specific ZmPIN1c probe showed that ZmPIN1c is localized in the epidermis and vasculature of the central cylinder (Figures $5 \mathbf{E}-\mathbf{G}$ ).

The localization pattern of the three ZmPIN1 genes was also confirmed by immunolocalization experiments, using an antiAtPIN1 antibody, which was shown to recognize the proteins of the three maize PIN1 genes expressed in the root. PIN1 proteins are polarly localized in the epidermis, meristem region, and central cylinder, where they are inserted in the basal cell membrane (Figure 5P) while they are detectable in the cytoplasm of the calyptrogens and proliferating cap (Figure 5Q). At protein level, the localization pattern of ZmPIN1a protein was observed using a ZmPIN1a-YFP reporter line (Gallavotti et al., 2008). At the confocal microscope the YFP signal was evident in the basal cell membranes of the central cylinder cells and in the cytoplasm of root cap cells and meristematic region of the root apex (Figures 5T-V and $\mathbf{7 H}$ ).

$Z m P I N 2$ transcripts were localized in root epidermis, exodermis, and multiple layers of cortex in cross section of the root apex (Figures 5H-M). Finally, ZmPIN9 the monocot-specific PIN gene is mainly expressed in endodermis, pericycle, and in phloem of the central cylinder (Figures 5N,O).

To correlate the localization pattern of the PIN genes expressed in maize root with auxin distribution and maximum in the same tissues, observations at the confocal microscope of a DR5::mRFP reporter line (Gallavotti et al., 2008) and immunolocalizations with an anti-IAA antibody on root longitudinal sections were both performed. Auxin response was observed in the root cap and vasculature in the DR5-RFP reporter line fresh root dissected at the confocal microscope (Figures 5W-Y and 7A,B). Auxin accumulation was evident in the root cap, in particular in the calyptrogen, in the epidermis at the level of root meristem and vasculature in longitudinal root sections labeled with the anti-IAA antibody (Figures 5R-S).

\section{ZmPIN GENES EXPRESSION ANALYSIS IN ROOTS OF B73 LINE AND br2 MUTANT}

The br2 maize mutant exhibits reduced shootward auxin transport at the root apex and reduced root gravitropic growth (McLamore et al., 2010). To investigate whether this alteration in auxin fluxes influenced the expression of $Z m P I N$ transporters, the mRNA levels of ZmPIN1a, ZmPIN1b, ZmPIN1c, ZmPIN2, and ZmPIN9 genes, the PIN members expressed in root apex, were analyzed by Real-Time PCR in different segments of B73 and br2 young primary roots.

As shown in Figure 6B, in $b r 2$ mutant the expression level of $Z m A B C B 1 / B R 2$ transcript was drastically reduced in all root segments (from A to E) compared to the levels observed in B73 line. 


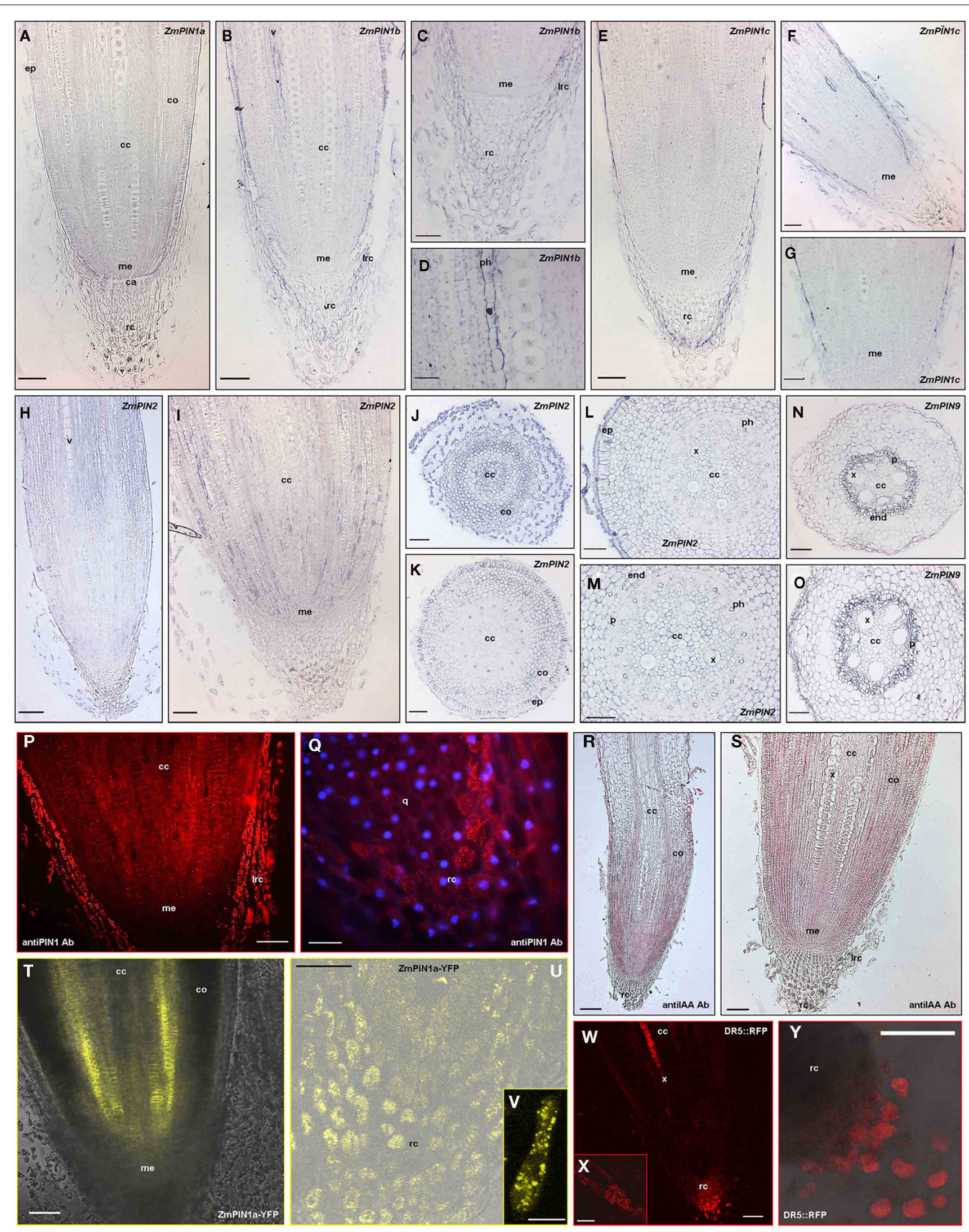

FIGURE 5 | Continued 


\author{
FIGURE 5 | Continued \\ Localization of PIN transporters and auxin maximum in maize root. \\ In situ hybridization on maize root sections with DIG-labeled antisense \\ probes of maize PIN transporters expressed in the root (A-P). The \\ hybridization signal is represented by the blue staining. Images were \\ acquired with a Leica DC300F camera. ZmPIN1 protein immunolocalization \\ $(\mathbf{Q}, \mathbf{R})$ and ZmPIN1a::YFP reporter line observation at the confocal \\ microscope $(\mathbf{U}, \mathbf{V})$ in maize primary root can be correlated with auxin \\ accumulation revealed by DR5 activity $(\mathbf{W}, \mathbf{X})$ or IAA immunolocalization \\ textbf(S-T). (A) Longitudinal section of primary root hybridized with the \\ ZmPIN1a antisense transcript; the hybridization signal is localized in the \\ root cap, particularly in the calyptrogen and root meristematic region. \\ (B-D) Longitudinal sections through a primary maize root hybridized with \\ ZmPIN1b antisense transcript showing the hybridization signal in root cap, \\ epidermis, endodermis, and vasculature of the central cylinder. In (B) the \\ signal is visible in root cap, epidermis, and vasculature. (C) portrays a \\ magnification of the root cap and (D) is a higher magnification of (B) \\ showing the blue staining in the phloem cells. (E-G) Longitudinal sections \\ of a maize primary root hybridized with a ZmPIN1C antisense probe: the \\ signal is detectable in root cap, epidermis, and vasculature. (H-M) In situ \\ hybridization on maize primary root sections with a ZmPIN2 DIG-labeled \\ antisense RNA probe. $(\mathbf{H}, \mathbf{I})$ are longitudinal sections of a primary root \\ showing the hybridization signal of ZmPIN2 in root cortex and central \\ cylinder. (J-M) depict cross sections through a maize primary root \\ hybridized with the ZmPIN2 antisense probe. (J) The picture portrays a \\ cross section of a root tip at the level of proximal root meristem in which \\ the signal is visible in the proliferating cells of the root cap, cortex, and \\ differentiating central cylinder. (K-M) Cross sections showing the \\ concentric tissues composing the maize primary root: epidermis,
}

exodermis, cortex, endodermis, xylem elements, phloem, and pericycle. In (K) the hybridization signal of ZmPIN2 is detectable in exodermis, cortex, and phloem. (L,M) show the hybridization signal in the cortex and phloem. (M) is higher magnifications of (L). ( $\mathbf{N}, \mathbf{O})$ Cross sections of a maize primary root hybridized with ZmPIN9 antisense riboprobe. The signal is localized in epidermis, exodermis, endodermis, and pericycle that surround the central cylinder. $(\mathbf{P}, \mathbf{0})$ Anti-PIN1 immunolocalization on longitudinal sections root apexes. The anti-AtPIN1 primary antibody shows protein localization on basal cell membranes of root meristematic region $(\mathbf{P})$ and in proliferating root cap $(\mathbf{P}, \mathbf{Q})$. DAPI was used to counterstain the nuclei in (Q). Both panels represent epifluorescence images acquired with a Leica DC300F camera. (R,S) Auxin immunolocalization in maize primary roots. In these longitudinal sections an anti-IAA monoclonal antibody shows a gradient of auxin toward the root tip, with the concentration maximum in the lateral root cap and in calyptrogens cells. (T-V) Confocal images of the pZmPIN1a::ZmPIN1a:YFP transgenic line root apex. ZmPIN1a-YFP is localized in proliferating root cap (U), in calyptrogens $(\mathbf{U})$, in the quiescent center $\mathbf{( T )}$, in the proximal root meristem $(\mathbf{T})$ and in the basal membranes of central cylinder cells (T). The single root cap cell in (V) shows the cytosolic localization of YFP signal in the root cap cells. (W-Y) Confocal images of a DR5::mRFP maize reporter line. Auxin accumulation is indirectly visualized in the tip of the root cap $(\mathbf{W}, \mathbf{Y})$ and in the vasculature of the central cylinder $(\mathbf{W})$. The inset $(\mathbf{X})$ represents a single xilematic cell showing DR5 activity while (Y) depicts details of (W). ca, calyptrogen; cc, central cylinder; co, cortex; ep, epidermis, end, endodermis; ex, exodermis; me, root meristem; p, pericycle; ph, phloem; q, quiescent center; rc, root cap; v, vasculature; $x$, xylem. Scale Bars: $200 \mu \mathrm{m}$ in $(\mathbf{H}, \mathbf{R})$; $150 \mu \mathrm{m}$ in (W); $100 \mu \mathrm{m}$ in (A-C,E-G,I-P,S,T,Y); $75 \mu \mathrm{m}$ in (U); $50 \mu \mathrm{m}$ in (D,Q,X); and $20 \mu \mathrm{m}$ in (V).
ZmPIN1a transcript showed a slight higher expression in the root segments A-C and lower levels in segments D-E of br2 mutant when compared with the same segments of B73. On the contrary, the mRNA level of $Z m P I N 1 b$ was higher in A-B-C root segments of br2 mutant, while in the D-E segments its level was comparable to that observed in wt. Also $Z m P I N 1 c$ showed a different expression pattern in $b r 2$ compared to B73 line: in particular, an up-regulation was observed in segment $A$, while a down-regulation occurs in segment D.

The transcript level of ZmPIN2 was slightly higher in all tested segment of $b r 2$ mutant, in particular in the distal region of the root (segments D and E) where PIN2 is not detectable in B73. Finally, ZmPIN9 transcript was up-regulated in segments C-D of br2 mutant.

Taken all together these results highlight that the expression profile of PIN transporters is altered in br2 mutant primary roots.

\section{GENE EXPRESSION ANALYSIS IN ROOTS OF B73 LINE AND br2 MUTANT UNDER DRUG TREATMENTS}

The expression profile of $Z m P I N$ genes and $Z m A B C B 1 / B R 2$ was investigated by Real-Time PCR in root apex of B73 and br2 mutant line grown in water (control), in presence of exogenous auxin (NAA), and of a PAT inhibitor (NPA; Figure 6C). Due to the strong reduction of root elongation caused by NAA application, the analysis was done just on the first two root segments indicated with A (from 0 to $0.2 \mathrm{~cm}$ ) and B (from 0.2 to $0.5 \mathrm{~cm}$ ).

In both lines the addition of NAA and NPA affected the expression of $Z m P I N$ genes and $Z m A B C B 1 / B R 2$ in both root segments. In particular in $\mathrm{B} 73, \mathrm{NPA}$ strongly inhibited $Z m A B C B 1 / B R 2$ expression in segment A, while NAA application doubled its expression levels in segment B. The same NAA effect was observed in $b r 2$ mutant roots. Both NAA and NPA treatments increased $\mathrm{ZmPINa}$ and $\mathrm{ZmPIN1c}$ transcript levels in segment B in both genotypes; on the contrary NAA application strongly reduced $Z m P I N 1 c$ levels in the segment A of br2 root. Similarly, ZmPIN1b transcript levels were strongly reduced after NAA application in br2 mutant roots (segments A and B), while NPA affected its expression exclusively in the B segment. NAA treatment increased ZmPIN9 transcript levels in the segment B of mutant roots, while ZmPIN2 expression was affected in a lower extent by the drug applications.

Experimental evidences showed that auxin transporter expression is altered by exogenous auxin or PAT inhibitor applications and these alterations are more dramatic in the br2 mutant background.

\section{DRUG TREATMENTS ALTER MORPHOLOGY, AUXIN DISTRIBUTION, AND ZmPIN1a-YFP LOCALIZATION IN PRIMARY ROOTS}

The effects of NAA and NPA applications were investigated at cellular level by microscope observation of DR5::mRFP and ZmPIN1a-YFP reporter lines (Figure 7). NAA applications caused the main effects on root anatomy, auxin responses, and PIN1a localization (Figures 7B,E,F,I,K). Exogenous auxin application indeed resulted in a size reduction of both root cap and meristem (compare Figures 7B,D) and in the formation of a pluristratified epidermis (Figures 7E,F). After the treatment, DR5 activity is diffused in the lateral root cap cells (Figure 7B) and not limited to the root cap tip as in the control (Figure 7D). Auxin response was detectable in the vasculature that differentiates very close to the root meristem (Figures 7B,E) and also the pluristratified epidermis showed DR5 activity (Figure 7F). Although PIN1a-YFP always localized in the stele cells in both NAA-treated and control 


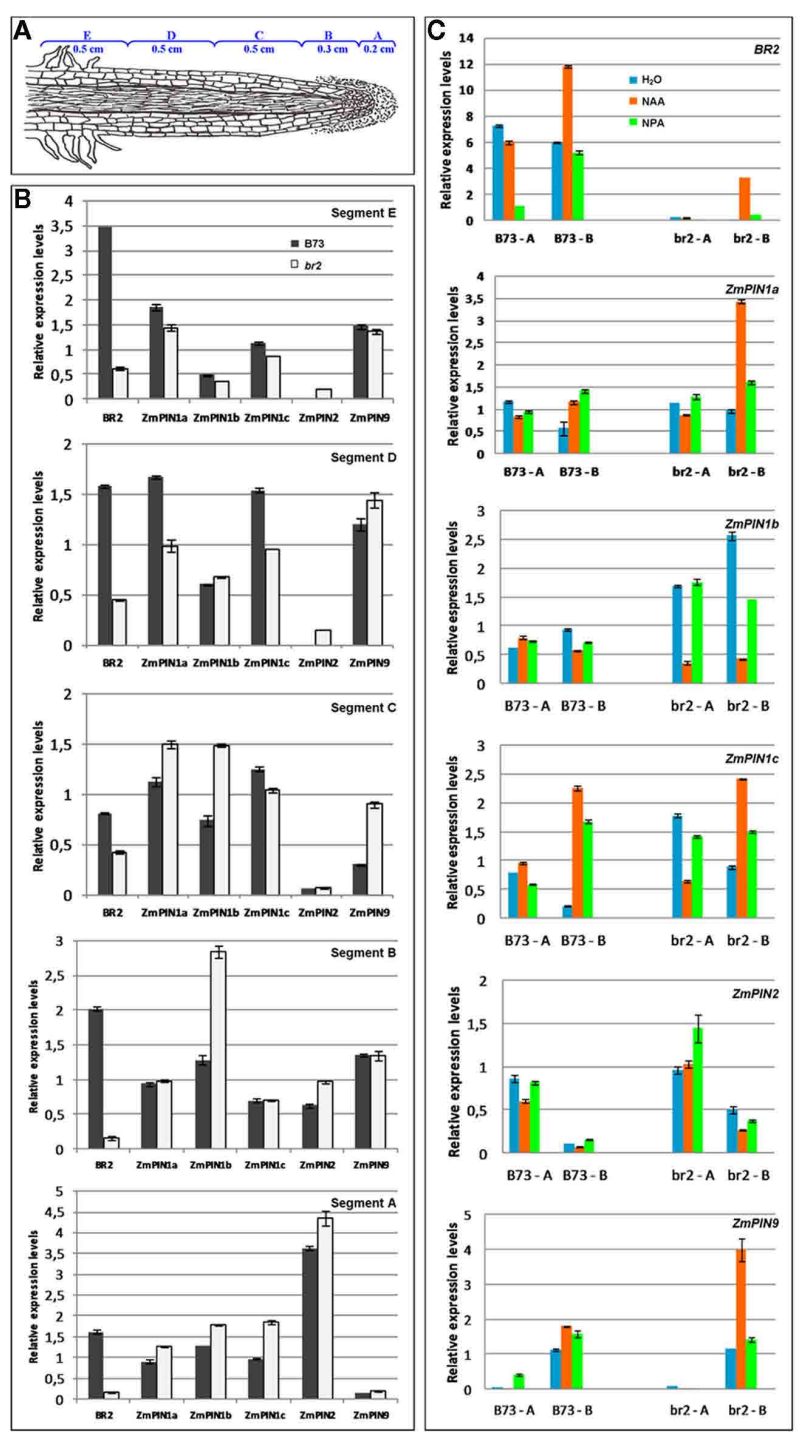

FIGURE 6 | br2/abcb1 mutation and drug applications alter auxin transporter expression levels. Quantitative RT-PCR analysis of $Z m A B C B 1 / B R 2, Z m P I N 1 a, Z m P I N 1 b, Z m P I N 1 c, Z m P I N 2$, and ZmPIN9 was performed in root segments (from A-E) of B73 line and br2 mutant under different treatments. The average value from three replicates and two biological samples was estimated by the generation of standard curves for each gene. Expression levels of each auxin transporter gene were normalized to GAPC2 transcript quantities. Standard deviation bars are indicated in the diagram. (A) Schematic representation of a maize root indicating the different segments used in expression analysis. (B) Quantitative RT-PCR expression analysis of auxin transporters in B73 and br2 mutant roots. The altered auxin shootward transport in the mutant root results in different expression levels of PIN mRNAs. (C) Quantitative RT-PCR expression analysis of auxin transporters after NAA and NPA application in B73 and br2 mutant roots. Applications of $50 \mu \mathrm{M}$ NAA and $50 \mu \mathrm{M}$ NPA to young seedlings result in a tissue-specific modulation of PIN transcripts. The most dramatic effects are observable in the mutant roots.

roots, some observed differences in protein localization reflected the changes in root morphology, particularly at the level of the vasculature and meristem (Figures 7H,I,K).
Following NPA applications, auxin signal was detectable in root cap as in the control (data not shown) while a diffuse DR5 activity marked the whole central cylinder and the cortex cells (Figures 7C,G). This suggests that NPA caused a failure in creating the auxin maximum detectable in the vascular cell in the control roots (Figure 7A). Similarly, the ZmPIN1a-YFP reporter line showed a more diffuse localization of the PIN1a protein that marked also the cortex cells (Figures 7J,L).

\section{ZmPIN LOCALIZATION PATTERN IN MAIZE INFLORESCENCES}

In maize, at the transition from vegetative to reproductive development the shoot apical meristem (SAM) becomes an inflorescence meristem (IM) which starts to produce lateral meristems called branch meristems (BMs). BMs develop spikelet-pair meristems (SPMs), each bearing two spikelet meristems (SMs). SMs form two floral meristems (FMs), the upper FM and lower FM, which produce the floral organ of the tassel. In each flower of the tassel, after gynoecial development, the gynoecium degenerates, and an imperfect staminate flower results. Within a few weeks after the SAM transition, a lateral shoot meristem in the axil of a leaf becomes an ear IM and produces multiple rows of SPMs, which follow the same step as in the tassel until the lower floret aborts as do the stamens of the upper floret. Indeed, in the mature ear a pistillate flower is present per spikelet (see McSteen et al., 2000 for a review on maize inflorescence development). Since ZmPIN1d transcript is specifically up-regulated in both male and female inflorescences, in situ hybridizations were performed in the SAM, starting from the transition stage to SPMs and spikelet differentiation, in male inflorescences, and during female inflorescence development (Figures 8A-H). During flowering transition, which is characterized by the elongation of the SAM and the adoption of an inflorescence identity to form a tassel primordium, ZmPIN1d hybridization signal is detectable in the L1 layer of the IM apical meristem (Figure 8A); later on, ZmPIN1d is localized in the L1 layer of the developing tassel SPMs primordium (data not shown). In the ear, ZmPIN1d showed the same localization patterns as in the tassel, being localized in the L1 layer of the IM during the transition phase and maintaining its localization in the L1 cell layer during SPM differentiation (Figures 8B,C). During SMs differentiation and growth, $Z m P I N 1 d$ is localized in the tips of the developing glumes (Figure 8D). Later on in ear development, during the pistillate flower differentiation, ZmPIN1d hybridization signal is detectable in the outer cell layers of the gynoecium, till the differentiation of the gynoecium ridge that will encircle the ovule region giving rise to the stylar canal (Figures $\mathbf{8 E}-\mathbf{G}$ ) and in the nucellus surrounding the mother cells of the megaspore (Figure 8H). The same localization pattern was observed in the pistillate flowers of female inflorescence sections hybridized with ZmPIN1a (Figures 8I-K), ZmPIN1b (Figure 8L), and ZmPIN2 (Figures 8M,N) antisense DIG-labeled mRNA probes.

\section{ZmPIN LOCALIZATION PATTERN IN MAIZE KERNEL}

RT-PCR showed that ZmPIN2, ZmPIN5c, ZmPIN8, and $Z m P I N 10 a$ are up-regulated during the early phases of kernel development, from 3 to 12 DAP. To precisely determine the localization of these genes at tissue level, in situ hybridization experiments were done on longitudinal sections of maize kernels from 5 


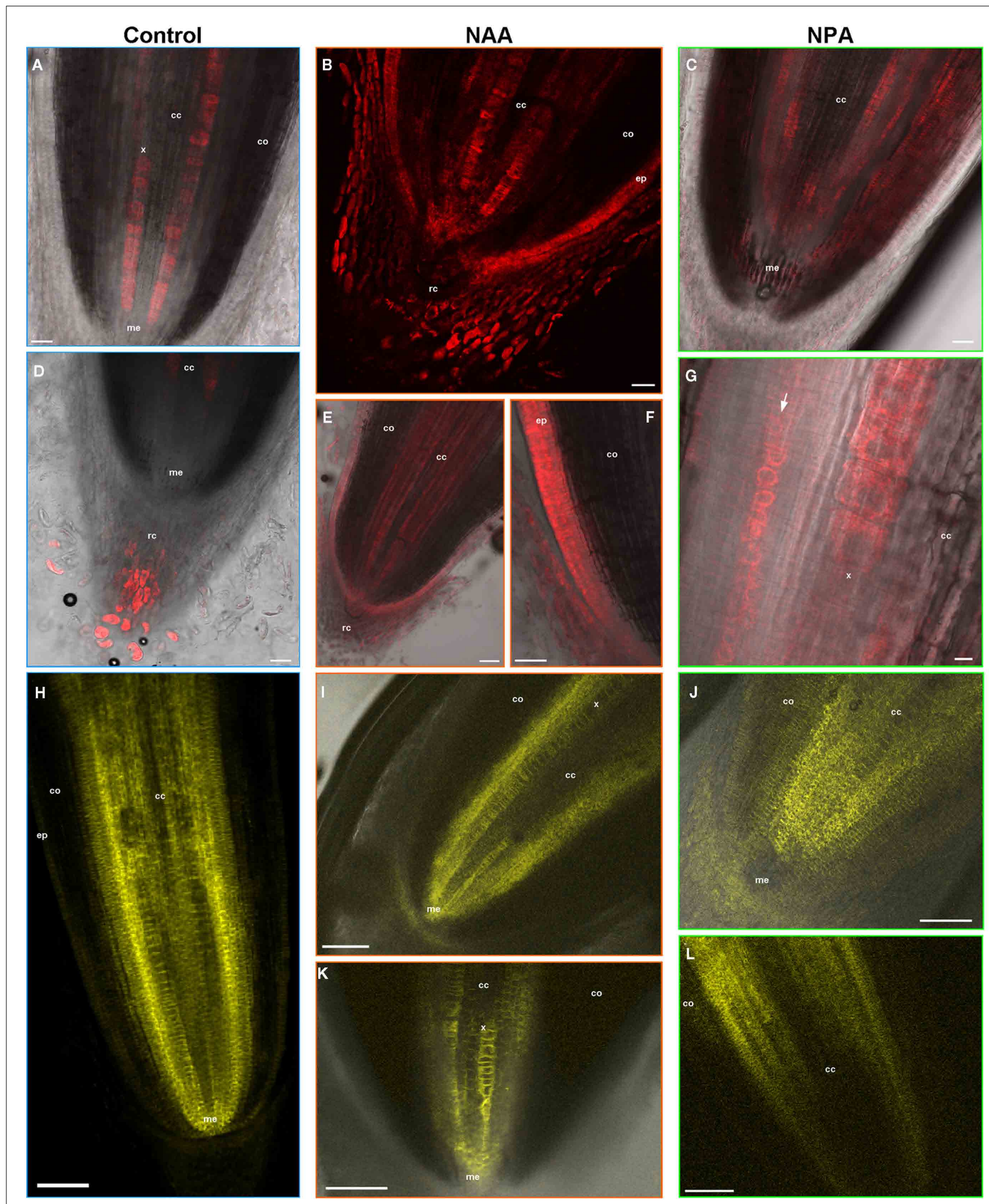




\section{FIGURE 7 | Continued \\ NAA and NPA applications alter root anatomy. auxin response and \\ PIN1a-YFP localization. The effects of NAA and NPA treatments on root anatomy, auxin gradients, and PIN1a protein localization are visualized by DR5:mRFP (A-G) and ZmPIN1a::YFP reporter lines observation at the confocal microscope (H-L). (A,D) Confocal images of a DR5::mRFP reporter line root grown in water. DR5 activity is visualized in the tip of the root cap (D) and in the vasculature of the central cylinder (A). (B,E,F) Exogenous auxin application results in root anatomic alteration (B,F) and in a diffused DR5 signal in the lateral root cap cells (B), in the central cylinder $(\mathbf{B}, \mathbf{E})$ and in the pluristratified epidermis (F). (C,G) Following NPA applications a diffuse DR5}

activity marks the whole central cylinder and the cortex cells. In particular high DR5 signal is visible in the endodermis cells [arrow in (G)]. (H) Confocal image of the pZmPIN1a::ZmPIN1a:YFP transgenic line root apex. ZmPIN1a-YFP is localized in the quiescent center, in the proximal root meristem and in the basal membranes of central cylinder cells. (I,K) PIN1a-YFP localization alteration in NAA-treated primary roots reflects the modified root anatomy at the level of the meristem and vascular tissues. (J,L) After NPA treatment ZmPIN1a-YFP reporter line shows a more diffuse signal of the PIN1a protein that marks also the cortex cells. cc, central cylinder; $c 0$, cortex; ep, epidermis, me, root meristem; rc, root cap; $x$, xylem. Scale Bars: $150 \mu \mathrm{m}$ in (H); $100 \mu \mathrm{m}$ in (E,I-L); $50 \mu \mathrm{m}$ in (A-D and $\mathbf{F})$ and $10 \mu \mathrm{m}$ in (G). to 12 DAP (Figure 9). During this early stage of maize kernel development the main cellular domains of the endosperm, the Basal Transfer Layer (BETL), the embryo surrounding region (ESR), the aleurone, and sub-aleurone differentiate, before the accumulation of reserves occurs. At 5 DAP ZmPIN2 is expressed in the differentiating endosperm ESR and in the embryo proper (Figure 9B); at 12 DAP later on during kernel development when the main cellular domains of the endosperm are completely differentiated, ZmPIN2 transcript is localized in the BETL, ESR, aleurone, and developing embryo at the transition stage (Figures 9A,C,K). ZmPIN5c transcripts are localized in ESR, BETL, and maternal chalazal cells of the kernel and in the embryo at both 5 and 12 DAP (Figures 9D-G); at 12 DAP its hybridization signal appears particularly evident in the outer epidermic layer of the embryo scutellum (Figure 9F), in the transition zone between BETL and aleurone (Figure 9G), while transcripts are not detectable in the aleurone layer (Figure 9L). ZmPIN10a transcript is also localized in both BETL and ESR endosperm cellular domains and embryo starting at 5 DAP (Figures 9H-I), and are also detectable in the chalazal and pedicel kernel tissues at 12 DAP (Figure 9H). Finally, ZmPIN8 is expressed in BETL cells, maternal chalazal tissues (Figure 9J), and aleurone layer (Figure 9M).

\section{DISCUSSION}

This work reports the identification and characterization of the $P I N$ auxin efflux carrier family members in $Z$. mays. In addition to the previously characterized ZmPIN1a, ZmPIN1b, and ZmPIN1c (Carraro et al., 2006; Forestan et al., 2010) we identified nine new members of the family, that is lastly made up by four genes homologous to Arabidopsis PIN1, three to AtPIN5, a PIN2, and a PIN8 ortholog and by three monocot-specific PINs (ZmPIN9, $Z m P I N 10 a$, and $Z m P I N 10 b)$. Anyway, more efforts are necessary to amplify and characterize the full-length CDSs of ZmPIN5b and ZmPIN10b, excluding (or confirming) the possibility they represent two pseudogenes.

Genes homologous to the Arabidopsis PINs are present in genomes throughout the plant kingdom, from the model moss $P$. patens to all vascular plants, and the relatively high amino acid identity between PIN proteins suggests that all the PIN genes diverged from a single ancestral sequence. At least four independent phylogenetic studies of PIN sequences from different plant species, including early-land plants, monocots, and dicots, revealed that the monocot PIN family is wider and more divergent than the dicots one, with two or three genes homologous to one single Arabidopsis PIN gene and also with the presence of at least one monocot-specific PIN gene (Paponov et al., 2005; Xu et al., 2005; Zazimalova et al., 2007; Krecek et al., 2009; Wang et al., 2009).

Our results strongly confirmed this hypothesis, showing a widening of PIN auxin efflux carriers family in maize, and more in general in monocots. By now it is largely accepted that maize genome undergoes a whole genome duplication (Helentjaris et al., 1988; Gaut and Doebley, 1997; Schnable et al., 2009) and several maize genes were found in duplicated chromosomic regions (Bomblies et al., 2003; Nardmann et al., 2004; Nardmann and Werr, 2006). Anyway, the widening of the monocots PIN family cannot be explained only by whole genome duplication, since in the diploid B. distachyon only two PINs (a PIN1 and a PIN5 ortholog) are missing compared to maize and rice. On the contrary, a whole genome duplication event could explain the widening of PIN members belonging to the P. trichocarpa family (Tuskan et al., 2006).

In detail, of the four PIN1 maize orthologs, chromosomal duplication analysis revealed that ZmPIN1a, ZmPIN1b, and $Z m P I N 1 c$ are located on duplicated regions on chromosomes 9, 5, and 4, respectively (Figures 2B,C and Figure S4 in Supplementary Material), and, together with ZmPIN1d share a common exon/intron structure (six exons and five introns, Figure 2A). These four genes encode for canonical long PIN proteins, with two conserved hydrophobic domains at the $\mathrm{N}$ - and C-termini and a long variable hydrophilic central region. Four PIN1 genes have also been found in rice (Wang et al., 2009): subfunctionalization of monocots PIN1 genes is confirmed by the expression analysis in different maize and rice plant tissues. ZmPIN1 $a$ and $Z m P I N 1 b$ are ubiquitously expressed, $Z m P I N 1 c$ is preferentially expressed in the post-embryonic root and stem while ZmPIN1d shows a high expression specificity, marking the transition from the vegetative to the reproductive development in SAM and IMs. Similarly in rice, OsPIN1 $a$ and OsPIN1b showed a constitutive expression, OsPIN1c is mainly expressed in root and stem while the expression pattern of OsPIN1d is still awaiting characterization (Wang et al., 2009).

A certain degree of expression specificity can be observed also analyzing maize PIN1 gene localization in the post-embryonic root: $Z m P I N 1 a, Z m P I N 1 b$, and $Z m P I N 1 c$ are expressed in root cap, epidermis, and central cylinder. However $Z m P I N 1 b$ is expressed in the lateral root cap and in the epidermis, ZmPIN1c is mainly detectable in the root cap tip and in the differentiating vasculature and $Z m P I N 1 a$ in the calyptrogens and in the root meristem. The specific ZmPIN1a transcript localization in root calyptrogen was confirmed at protein level by both immunolocalization experiments and observations of a ZmPIN1a:YFP reporter line. Both 


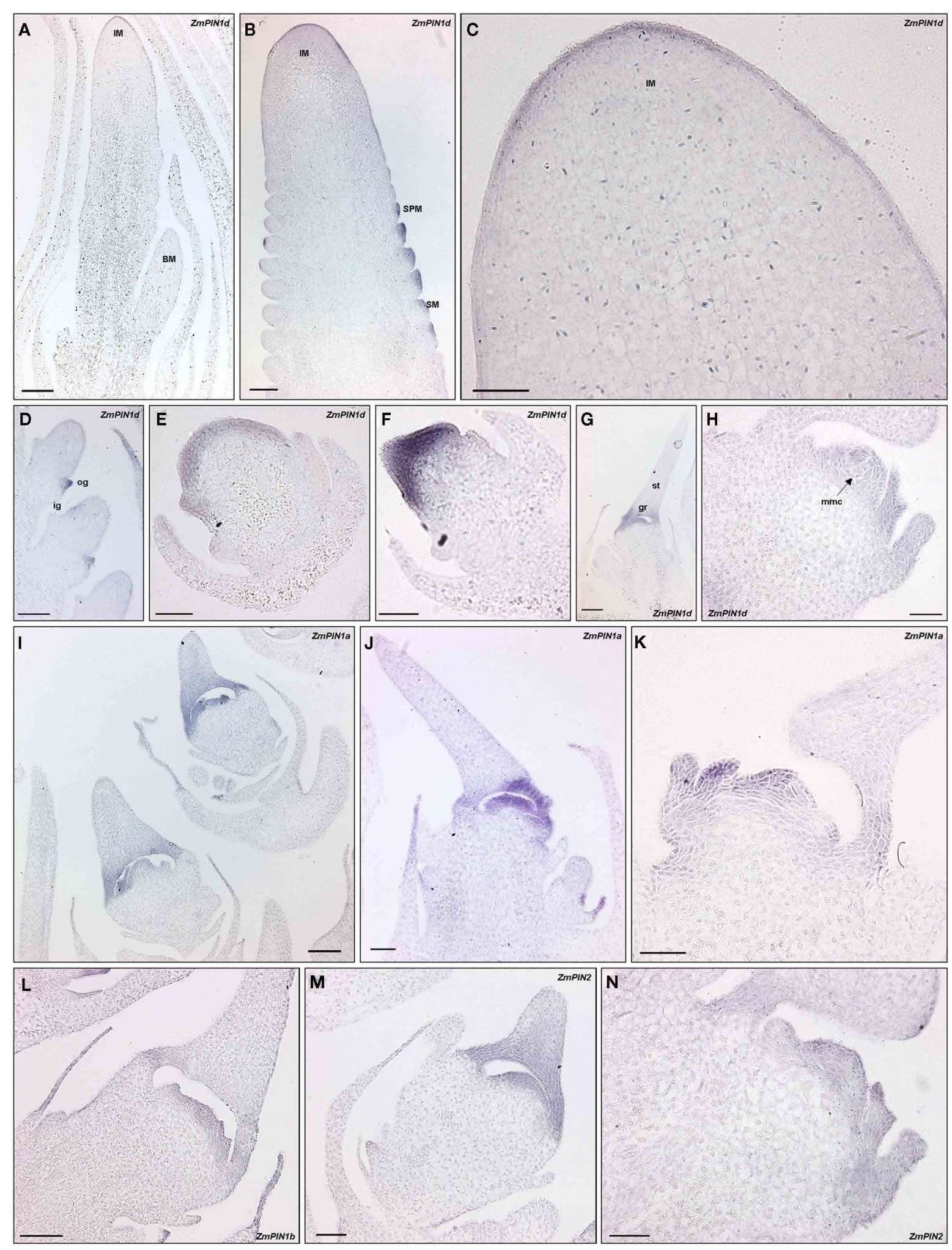




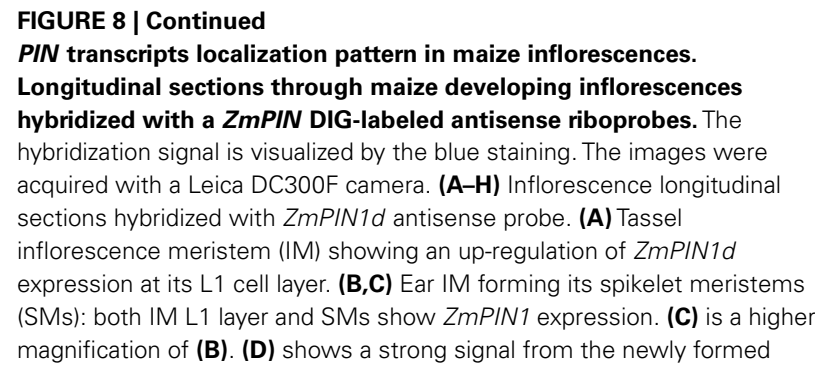

glume primordia of ear SMs. (E-H) Longitudinal sections of developing pistillate flowers. ZmPIN1d hybridization signal is detectable in the outer cell layers of the gynoecium till the differentiation of the gynoecium ridge that will encircle the ovule region giving rise to the stylar canal and in the nucellus surrounding the mother cells of the megaspore. (I-N) ZmPIN1a (I-K), ZmPIN1b (L), and ZmPIN2 (M-N) expression domains during the development of pistillate flowers overlap that of ZmPIN1d. IM, inflorescence meristem; SM, spikelet meristem; SPM, spikelet-pair meristem; ig, inner glume; og, outer glume; st, stylar canal; gr, gynoecium ridge; $\mathrm{mmc}$, megaspore mother cell. Scale Bars: $100 \mu \mathrm{m}$ in (A-B,D,G,I-J,L); $50 \mu \mathrm{m}$ in (C,E,F,H,K,M,N). localization procedures showed that PIN1 proteins are localized in the proximal root meristem. The anti-AtPIN1 antibody also shows the polarization of ZmPIN1 proteins in the cortex and central cylinder cells, suggesting that the proteins are involved in auxin transport toward root apex, as modeled for Arabidopsis root. Interestingly, in calyptrogen $\mathrm{ZmPIN} 1 \mathrm{a}$ is not inserted in cell plasma membrane but is accumulated in the cytoplasm. The same ZmPIN1 accumulation pattern was previously observed in the ESR during maize endosperm development (Forestan et al., 2010) and ZmPIN1a has been observed to accumulate in intracellular compartments also by the transformation of tobacco protoplast with a ZmPIN1a::GFP fusion construct (Forestan and Varotto, 2010). Interestingly, in maize endosperm ESR the accumulation of PIN1 proteins in the cell endomembranes is coupled with an auxin maximum in the same cells. Similarly in maize root, both anti-IAA immunolocalization and observation of a DR5-reporter line showed the presence of an auxin maximum in the root cap.

An auxin accumulation also characterizes the Arabidopsis root cap and columella cells (Sabatini et al., 1999): auxin is supplied to the root cap by the PIN1- and PIN4-dependent acropetal route and then laterally redistributed by PIN3- and PIN7-mediated efflux from columella cells (reviewed in Peer et al., 2011). The simultaneous and coordinated expression of ZmPIN1a, ZmPIN1b, and $Z m P I N 1 c$ in the maize root apex could suggest that these three proteins play the role of the Arabidopsis PIN3, PIN4, and PIN7 efflux carriers. Indeed, as in rice, no ZmPIN genes were grouped into the AtPIN3, AtPIN4, or AtPIN7 cluster (Wang et al., 2009). A previous phylogenetic analysis suggested a close evolutionary relationship between these three Arabidopsis transporters: AtPIN3 and AtPIN7 are located on two copies of a duplicated block of 285 genes on chromosome 1 and the rate of synonymous substitution analysis indicates that these blocks are the result of a recent duplication. Similarly, AtPIN3 and AtPIN4 share the same relative position in duplicated blocks of genes. However, these blocks are too small to be considered as statistically significant (Paponov et al., 2005). Our Phylip-phylogenetic study seems to suggest an evolutionary relationship between the AtPIN3/AtPIN4/AtPIN7 cluster and PIN10a/PIN10b monocots proteins that belong to a monophyletic group. However, this hypothesis is not confirmed by the evolutionary analysis based on Maximum Likelihood method, making any speculation weak. Nor can the expression analysis of the two maize PIN10 genes support this evidence: $Z m P I N 10 a$ is expressed in leaves and kernels and both ZmPIN10a and ZmPIN10b are expressed in male inflorescence at the limit of detection, as confirmed by a search in Genome-Wide Expression Atlas (Figure S5 in
Supplementary Material; Sekhon et al., 2011), with an expression pattern totally different from AtPIN3, AtPIN4, and AtPIN7.

Although the diploid monocot $B$. distachyon has only two PIN5 genes (the transporters lacking the central hydrophilic domain), rice, sorghum, and maize have three PIN5 sequences in their genome. In maize there is no evidence of duplication events involving regions in which PIN5 genes map; anyway both the divergent expression pattern of the three genes and the putative pseudogene nature of $Z m P I N 5 b$ suggest that the tracks of any potential duplication event might have been lost during chromosomal rearrangements and the subfunctionalization of these sequences.

In addition to PIN5s, a fourth short PIN transporter has been identified as the AtPIN8 ortholog and named ZmPIN8. All the short PIN transporters, the three PIN5 and PIN8 proteins, have a conserved hydrophobic profile with their orthologs in Arabidopsis that are localized in ER. However, this peculiar subcellular localization, which has been suggested as having an important implication from an evolutionary point of view (Wabnik et al., 2011), is still awaiting confirmation in monocots.

The monocot-specific ZmPIN9 and OsPIN9 proteins do not correspond to the traditional classification in long and short PINs, based on the length of hydrophilic domain. Their transcripts are specifically localized in the root and their proteins show an intermediate length of the hydrophilic domain. Furthermore, they grouped (together with sorghum and Brachypodium orthologs) in the same cluster with the P. patens PIND, whose intron sequences analysis suggested the possibility of its horizontal transfer from monocots (Krecek et al., 2009; Mravec et al., 2009). Unlike PpPINA, PpPINB, and PpPINC, the three closely related Physcomitrella PINs, which were localized in ER in transient transformation assays using tobacco protoplasts, PpPIND protein showed a non-specific cytosolic localization pattern (Mravec et al., 2009). All these observations make the investigation on subcellular localization of monocot PIN9 proteins particularly interesting.

Although $Z m P I N 2$ gene was expressed in vegetative and reproductive tissues and during kernel development, its locus was not identified in the maize genome 5 b. 60 release. ZmPIN2 has a conserved intron/exon structure with AtPIN2 but, similarly to its ortholog in rice (Wang et al., 2009) it does not have the same specific expression pattern at the root level. $Z m P I N X$ gene is a second missing gene in the maize genome database and together with ZmPINY represents the first PIN-like sequences characterized in maize so far. As for Arabidopsis, phylogenetic analysis revealed that PIN and PIN-like families form distinct clusters (Figure S1 in Supplementary Material; Paponov et al., 2005). 


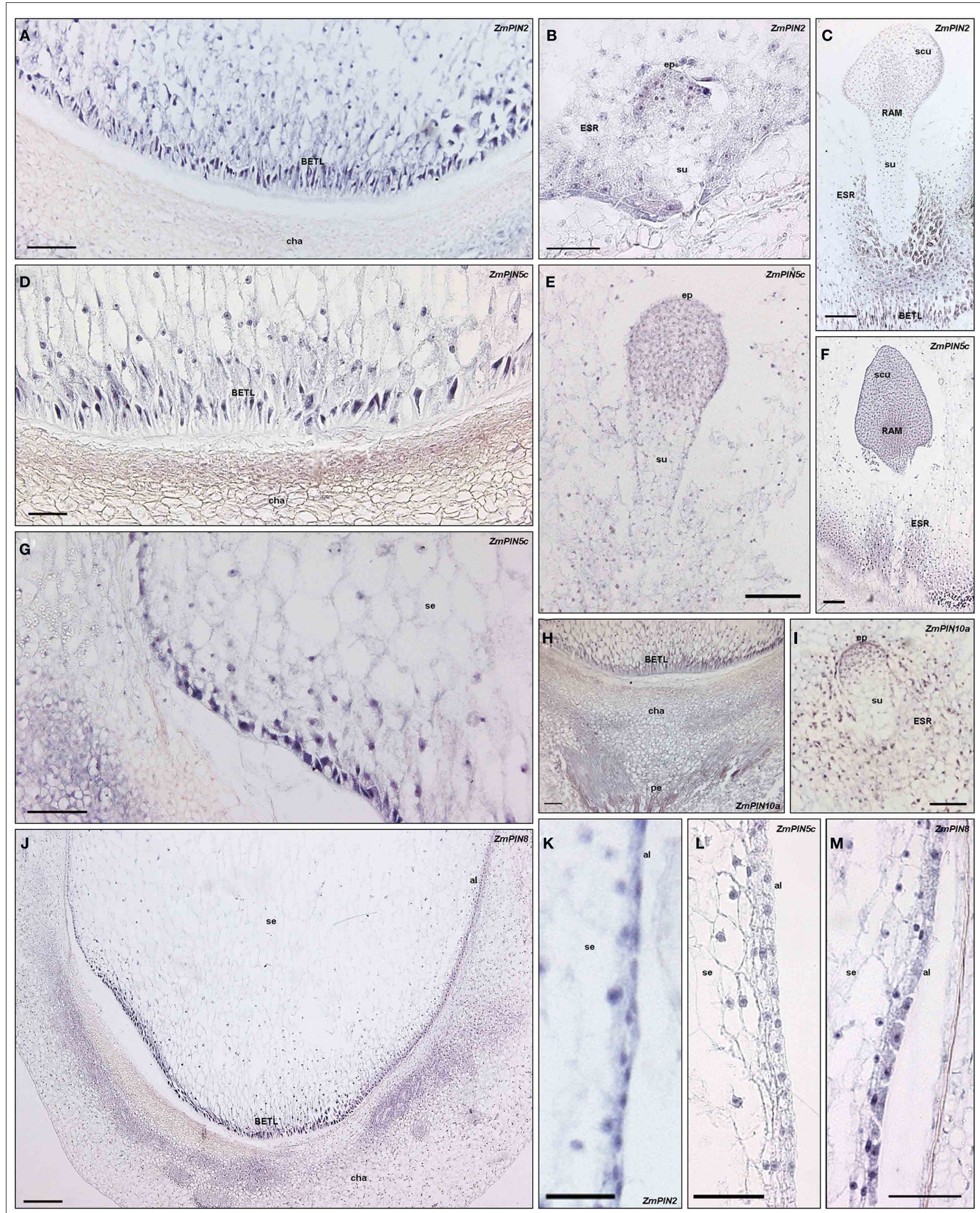




\begin{abstract}
FIGURE 9 | Continued
Localization pattern of PINs transporters in maize kernel. Longitudinal sections through maize kernels at different DAP, hybridized with antisense mRNA DIG-labeled probes corresponding to the PIN transporters expressed in maize caryopses. The hybridization signal is visualized by the blue staining (A-C) ZmPIN2 mRNA localization during kernel development. (A) In 12 DAP endosperm section ZmPIN2 transcripts localize in the basal endosperm transfer layers facing the maternal placenta of the seed. (B,C) Depict longitudinal section through two maize kernels at 6 and 12 DAP, respectively: in both sections, the hybridization signal is detectable in the endosperm embryo surrounding region (ESR) and in the embryo where it marks the outer cell layers. (D-G) ZmPIN5c transcript in situ hybridization in embryos and endosperm. (D,G) Show longitudinal sections through a 12 DAP kernel; ZmPIN5c hybridization signal is localized in the completely differentiated transfer cells facing the placenta in (D) and also in the differentiating cells at the flanks of the kernel, where the aleurone starts in (G). (E,F) Are longitudinal sections of maize kernel at 9 and 14 DAP, respectively, showing
\end{abstract}

ZmPIN5c hybridization signal in the embryo. In transition stage embryos ZmPIN5c marks the embryo proper, while no signal is detectable in the suspensor (E); later on development, in L1-stage embryo the transcripts localize in the scutellum and Root Apical Meristem (F). (H,I) ZmPIN10a antisense hybridizations in longitudinal sections of kernels and embryos. In 12 DAP kernels the hybridization signal is visible in both the BETL layer and maternal tissues (placenta and pedicel) of the seed $\mathbf{( H )}$, while at 5 DAP the ESR and the embryo proper are labeled (I). (J) ZmPIN8 mRNA localization in longitudinal section of a maize kernel at 12 DAP. The blue staining is detectable in the outer cell layers of the endosperm comprising the BETL and the aleurone. (K-M) ZmPIN2, ZmPIN5c, and ZmPIN8 expression in the aleurone layer at 12 DAP. Both ZmPIN2 and ZmPIN8 are highly expressed in the single cell layer of the aleurone (K,M), while no expression of ZmPIN5c is detectable in this domain (L). BETL, basal endosperm transfer layer; ESR, embryo surrounding region; cha, maternal chalazal region; pe, pedicel; se, starchy endosperm; al, aleurone; ep, embryo proper; su, suspensor; scu, scutellum. Scale bars: $200 \mu \mathrm{m}$ in (J); $100 \mu \mathrm{m}$ in (A-I); $50 \mu \mathrm{m}$ in (K-M).
Together with ZmPIN1a, ZmPIN1b, ZmPIN1c, ZmPIN2, and $Z m P I N 9, Z m A B C B 1 / B R 2$ is expressed in maize root apexes, particularly in root epidermis as previously reported (Knoller et al., 2010; McLamore et al., 2010). In br2 mutant root segments the expression levels of ZmPIN transcript are differentially altered, depending on the gene and segment. These observed fluctuations at transcript level can be correlated to the reduced auxin transport that characterizes br2 root (McLamore et al., 2010). This statement is confirmed by the expression analysis of ZmPIN genes in wt and $b r 2$ root treated with NAA and NPA. Indeed, also in B73 and $b r 2$ treated-root the expression levels of ZmPIN transcript were differentially altered, depending on the gene and segment. However, observing the ZmPIN1a and DR5-reporter lines, it became evident that both NAA and NPA treatments had a strong effect not only on auxin distribution but also on root tissues differentiation and ZmPINla protein localization. All together these evidences indicate that the NAA and NPA treatment (and may be also the br2 mutation), affect the root tissue differentiation indirectly changing the localization of the PIN transcripts and proteins, which appear to have a highly tissue-specific expression in maize root. Interestingly, the combination of $b r 2$ mutation and NAA or NPA treatment has additional effect on the alteration of ZmPIN gene expression levels.

This high tissue-specific regulation of PIN genes is a further indication that, in spite of a partial overlapping in their transcript localization in the root tissues, maize PIN genes might have acquired subfunctionalization during evolution.

Unlike in Arabidopsis, where each PIN has a specific expression domain, expression analysis revealed that many $Z m P I N$ genes are simultaneously expressed in the same organ or tissue. Indeed ZmPIN1a, ZmPIN1b, ZmPIN1d, and ZmPIN2 mark the development of the gynoecium and the formation of the silk in the female inflorescence, $Z m P I N 2, Z m P I N 5 c$, and ZmPIN8 are expressed, together with $Z m P I N 1 a, Z m P I N 1 b$, and ZmPIN1c, in the basal endosperm transfer layer (BETL) cells. These overlapping expression domains in different tissues and at different developmental stages indicate a great degree of functional redundancy among the members of maize PIN family. To understand the biological meaning behind this redundancy it is necessary to further investigate the different endogenous and exogenous stimuli able to regulate PIN gene expression and determine auxin gradients in the different plant tissues.

Another open point concerns auxin distribution in maize root apex: anti-IAA immunolocalization experiments and confocal images of a DR5-reporter line showed contrasting auxin distribution and accumulation patterns. It is well known that these two experimental approaches visualize free-auxin distribution and activity of auxin responsive promoter, respectively (Schlicht et al., 2006). Our results suggest that these approaches might be used together to better analyze auxin distribution and accumulation pattern in plant tissues.

In maize root apex auxin distribution and accumulation patterns differ from that reported for Arabidopsis, pointing out the importance of the root cap tip and epidermis in the creation of auxin gradients. Further investigations are needed to elucidate whether these differences in the localization of auxin gradients reflect the different anatomical organization between monocot and dicot roots or vice-versa.

\section{ACKNOWLEDGMENTS}

The authors thank N. Carraro and L. Ceccato for helpful discussion during the preparation of the manuscript, Prof. D. Jackson for providing us with the reporter lines, Dr. R. Pilu for the $b r 2$ mutant seeds and A. Garside for checking the English. Authors also thank the two reviewers for their useful comments and suggestions. The work represents the starting point of FIRB - Progetto Giovani of the Italian MIUR.

\section{SUPPLEMENTARY MATERIAL}

The Supplementary Material for this article can be found online at http://www.frontiersin.org/Plant_Traffic_and_Transport/10.3389/ fpls.2012.00016/abstract

\footnotetext{
Figure S1 | Neighbor-joining phylogenetic analysis of PIN auxin efflux carriers from Zea mays, Arabidopsis thaliana, Medicago truncatula, Oryza sativa, Brachypodium distachyon, Sorghum bicolor, Setaria italica, Populus trichocarpa, Vitis vinifera, Physcomitrella patens, and Selaginella moellendorffii. The phylogenetic analysis between newly identified maize PIN proteins, PIN and PIN-like proteins of eudicots and monocots, summarizes the evolutionary relationships among the 120 members of the PIN protein family.
} 
PIN protein sequences from two herbaceous eudicots ( $A$. thaliana, $A t$, and M. truncatula, Medtr), two arboreal eudicots ( $P$. trichocarpa, Ptri, and V. vinifera, Vvinifera), four monocots (O. sativa, Os; S. bicolor, Sb; B. distachyon, Bdist, and $S$. italica, Sitalica), a bryophyte (P. patens, Pp), and a lycophyte (S. moellendorffii,) were retrieved by family blast searches in the Phytozome $\mathbf{v} .0$ database (www.phytozome.net/) and were aligned together with newly identified ZmPIN and PIN-like proteins of maize. Alignment were performed with ClustalX 1.81 (Blosum Weight Matrix; Gap Opening Penalty: 5; Gap Extension Penalty: 0.20), and a neighbor-joining phylogenetic tree was prepared with Phylip package using 100 bootstraps. The yeast PIN-like protein AEL1 (NP_593365 from Schizosaccharomyces pombe) was included in the analysis as outgroup. Newly identified ZmPIN proteins are named according to the cluster of the Arabidopsis and rice PIN family to which they belong. Accession numbers are reported in Table S1.

Figure S2 | Phylogenetic network of PIN auxin efflux carriers from Zea mays, Arabidopsis thaliana, Oryza sativa, Brachypodium distachyon, Populus trichocarpa, Physcomitrella patens, and Selaginella moellendorffii. PIN protein sequences of $Z$. mays $(Z m), A$. thaliana $(A t), P$. trichocarpa (Ptri), $O$. sativa (Os), B. distachyon (Bdist), P. patens (Pp), and S. moellendorffii $(\mathrm{Sm})$ were retrieved by family blast searches in the Phytozome v7.0 database (www.phytozome.net/) and aligned with MAFFT algorithm available at the EMBL-EBI website (http://www.ebi.ac.uk/Tools/msa/mafft/). A NeighborNet Network was constructed with SplitsTree4 software. In comparison with the classical phylogenetic tree, this network-based approach allows a richer visualization of the data, taking in account that many events such as hybridization, horizontal gene transfer, recombination, or gene duplication and loss might be involved in evolution of the PIN family across plant species.

Figure S3 | Maximum Likelihood phylogenetic analysis of PIN auxin efflux carriers from Zea mays, Arabidopsis thaliana, Oryza sativa, Brachypodium distachyon, Populus trichocarpa, Physcomitrella patens, and Selaginella moellendorffii. PIN protein sequences of $Z$. mays $(Z m)$, $A$. thaliana $(A t), P$. trichocarpa (Ptri), O. sativa (Os), B. distachyon (Bdist), P. patens (Pp), and S. moellendorffii $(\mathrm{Sm})$ were retrieved by family blast searches in the Phytozome v7.0 database (www.phytozome.net/) and aligned with MAFFT algorithm available at the EMBL-EBI website (http://www.ebi.ac.uk/Tools/msa/mafft/). PIN proteins were aligned with MAFFT algorithm available at the EMBL-EBI website (http://www.ebi.ac.uk/Tools/msa/mafft/). Phylogeny was inferred with the Molecular Evolutionary Genetic Analysis 5 (MEGA5) program using the Maximum Likelihood method (10,000 bootstraps, Jones-Taylor-Thornton model).

Figure S4 | ZmPINs duplication analysis. The upper panel shows the duplicated regions between the 10 maize chromosomes. The map positions of the maize PIN genes have been manually reported in the scheme. In the bottom panel the duplications of ZmPIN1a-ZmPIN1b (on chromosomes 9 and 5,

\section{REFERENCES}

Avsian-Kretchmer, O., Cheng, J. C., Chen, L., Moctezuma, E., and Sung, Z. R. (2002). Indole acetic acid distribution coincides with vascular differentiation pattern during Arabidopsis leaf ontogeny. Plant Physiol. 130, 199-209.

Benkova, E., Michniewicz, M., Sauer, M., Teichmann, T., Seifertova, D., Jurgens, G., and Friml, J. (2003). Local, efflux-dependent auxin gradients as a common module for plant organ formation. Cell 115, 591-602.

Blakeslee, J. J., Bandyopadhyay, A., Peer, W. A., Makam, S. N., and Murphy, A. S. (2004). Relocalization of the PIN1 auxin efflux facilitator plays a role in phototropic responses. Plant Physiol. 134, 28-31.

respectively) and $Z m P I N 1 b-Z m P I N 1 c$ (on chromosomes 5 and 4, respectively) blocks is depicted. Finally, ZmPIN5a, ZmPIN9, ZmPIN8, and ZmPIN10a map on long arm of chromosome 3 , in a region highly syntenic to the rice chromosome 1, where OsPIN5a, OsPIN9, OsPIN8, and OsPIN10a map too. Duplicated and syntenic blocks have displayed with Synteny Mapping and Analysis Program (SyMAP) v3.4 (Soderlund et al., 2011; http://www.symapdb.org/).

Figure S5 | Maize auxin transporter expression analysis from the Genome-wide atlas of transcription during maize development. ZmPINs, $Z m P I N-l i k e$ and BR2/ZmABCB1 expression levels analyzed in 60 distinct tissues representing 11 major organ systems of inbred line B73 (Sekhon et al., 2011). Expression graphs have been download from www.maizegdb.org: the green bars indicate tissues in which the gene is expressed.

Figure S6 | In situ hybridization and anti-IAA negative controls. Specific sense RNA probes for each PIN genes were used as negative controls in every in situ hybridization experiment (A-D) while two different negative controls, no EDAC pre-fixation and without anti-IAA primary antibody were used. (A) Longitudinal section of a primary root hybridized with a ZmPIN1a sense probe. (B) Longitudinal section of a primary root apex hybridized with a ZmPIN1b sense probe. (C) Cross section of primary root hybridized with a ZmPIN9 sense probe. (D) Longitudinal section of a female inflorescences showing the mother cell of the megaspore inside the ovule hybridized with a ZmPIN1d sense probe. (E) Anti-IAA negative control on longitudinal section of a primary root not pre-fixed with EDAC. (F,G) Anti-IAA negative control on longitudinal sections of a primary root hybridized with the secondary antibody only. $\mathrm{cc}$, central cylinder; co, cortex; ep, epidermis, end, endodermis; me, root meristem; p, pericycle; ph, phloem; rc, root cap; $\mathrm{x}$, xylem; st, stylar canal; mmc, megaspore mother cell. Scale Bars: $200 \mu \mathrm{m}$ in (A); $100 \mu \mathrm{m}$ in (B-G).

Table S1 | PIN sequences accession numbers. Accession numbers list, common name (where available) and respectively protein sequences of auxin efflux carriers considered in this work for phylogenetic analysis are reported for each species. While Arabidopsis thaliana and Zea mays PIN and PIN-like sequences were retrieved from TAIR (www.arabidopsis.org) and MaizeGDB (www.maizegdb.org) databases, respectively, PIN protein sequences of the other species were identified by a family blast search in the Phytozome v7.0 database (www.phytozome.net/).

Table S2 | Primer list. Primers used for cDNA and genomic full-length amplification, amplification of probes for in situ hybridization and expression analysis in vegetative and reproductive tissues (with relative number of circles considered in each PCR) are listed for each maize PIN and PIN-like gene.

Table S3 | Maize PIN and PIN-like map position. Chromosomal position (obtained from www.maize.sequence.org database) of the newly identified maize PIN and PIN-like sequences.

encode two novel putative candidates for polar auxin transport and plant architecture determination of maize. Plant Physiol. 142, 254-264.

De Smet, I., and Jurgens, G. (2007). Patterning the axis in plants - auxin in control. Curr. Opin. Genet. Dev. 17, 337-343.

Dubrovsky, J. G., Sauer, M., NapsucialyMendivil, S., Ivanchenko, M. G., Friml, J., Shishkova, S., Celenza, J., and Benkova, E. (2008). Auxin acts as a local morphogenetic trigger to specify lateral root founder cells. Proc. Natl. Acad. Sci. U.S.A. 105, 8790-8794.

Ellis, C. M., Nagpal, P., Young, J. C., Hagen, G., Guilfoyle, T. J., and Reed, J. W. (2005). AUXIN RESPONSE
FACTOR1 and AUXIN RESPONSE FACTOR2 regulate senescence and floral organ abscission in Arabidopsis thaliana. Development 132, 4563-4574.

Felsenstein, J. (1989). PHYLIP - phylogeny inference package. Cladistics 5, 164-166

Forestan, C., Meda, S., and Varotto, S. (2010). ZmPIN1-mediated auxin transport is related to cellular differentiation during maize embryogenesis and endosperm development. Plant Physiol. 152, 1373-1390.

Forestan, C., and Varotto, S. (2010). PIN1 auxin efflux carriers localization studies in $\mathrm{Zea}$ mays. Plant Signal. Behav. 5, 436-439. 
Forestan, C., and Varotto, S. (inpress). The role of PIN auxin efflux carriers in polar auxin transport and accumulation and their effect on shaping maize development. Mol. Plant. doi: $10.1093 / \mathrm{mp} / \mathrm{ssr} 103$

Friml, J. (2010). Subcellular trafficking of PIN auxin efflux carriers in auxin transport. Eur. J. Cell Biol. 89, 231-235.

Friml, J., Vieten, A., Sauer, M., Weijers, D., Schwarz, H., Hamann, T., Offringa, R., and Jurgens, G. (2003). Efflux-dependent auxin gradients establish the apical-basal axis of Arabidopsis. Nature 426, 147-153.

Gallavotti, A., Yang, Y., Schmidt, R. J., and Jackson, D. (2008). The Relationship between auxin transport and maize branching. Plant Physiol. 147, 1913-1923.

Gaut, B. S., and Doebley, J. F. (1997). DNA sequence evidence for the segmental allotetraploid origin of maize. Proc. Natl. Acad. Sci. U.S.A. 94, 6809-6814.

Geisler, M., and Murphy, A. S. (2006). The ABC of auxin transport: the role of p-glycoproteins in plant development. FEBS Lett. 580, 1094-1102.

Guo, A. Y., Zhu, Q. H., Chen, X., and Luo, J. C. (2007). GSDS: a gene structure display server. Yi Chuan 29, 1023-1026.

Heisler, M. G., Ohno, C., Das, P., Sieber, P., Reddy, G. V., Long, J. A., and Meyerowitz, E. M. (2005). Patterns of auxin transport and gene expression during primordium development revealed by live imaging of the Arabidopsis inflorescence meristem. Curr. Biol. 15, 1899-1911.

Helentjaris, T., Weber, D., and Wright, S. (1988). Identification of the genomic locations of duplicate nucleotide sequences in maize by analysis of restriction fragment length polymorphisms. Genetics 118, 353-363.

Hochholdinger, F., Park, W. J., Sauer, M., and Woll, K. (2004). From weeds to crops: genetic analysis of root development in cereals. Trends Plant Sci. $9,42-48$.

Huson, D. H., and Bryant, D. (2006). Application of phylogenetic networks in evolutionary studies. Mol. Biol. Evol. 23, 254-267.

Katoh, K., Asimenos, G., and Toh, H. (2009). Multiple alignment of DNA sequences with MAFFT. Methods Mol. Biol. 537, 39-64.

Katoh, K., Misawa, K., Kuma, K., and Miyata, T. (2002). MAFFT: a novel method for rapid multiple sequence alignment based on fast Fourier transform. Nucleic Acids Res. 30, 3059-3066.
Kerr, I. D., and Bennett, M. J. (2007). New insight into the biochemical mechanisms regulating auxin transport in plants. Biochem. J. 401, 613-622.

Kimura, M., and Kagawa, T. (2006). Phototropin and light-signaling in phototropism. Curr. Opin. Plant Biol. 9, 503-508.

Knoller, A. S., Blakeslee, J. J., Richards, E. L., Peer, W. A., and Murphy, A. S. (2010). Brachytic2/ZmABCB1 functions in IAA export from intercalary meristems. J. Exp. Bot. 61, 3689-3696.

Krecek, P., Skupa, P., Libus, J., Naramoto, S., Tejos, R., Friml, J., and Zazimalova, E. (2009). The PINFORMED (PIN) protein family of auxin transporters. Genome Biol. 10, 249

Krogh, A., Larsson, B., von Heijne, G., and Sonnhammer, E. L. (2001). Predicting transmembrane protein topology with a hidden Markov model: application to complete genomes. J. Mol. Biol. 305, 567-580.

Leyser, O. (2005). The fall and rise of apical dominance. Curr. Opin. Genet. Dev. 15, 468-471.

McLamore, E. S., Diggs, A., Calvo Marzal, P., Shi, J., Blakeslee, J. J., Peer, W. A., Murphy, A. S., and Porterfield, D. M. (2010). Non-invasive quantification of endogenous root auxin transport using an integrated flux microsensor technique. Plant J. 63, 1004-1016.

McSteen, P., Laudencia-Chingcuanco, D., and Colasanti, J. (2000). A floret by any other name: control of meristem identity in maize. Trends Plant Sci. 5, 61-66.

Mravec, J., Skupa, P., Bailly, A., Hoyerova, K., Krecek, P., Bielach, A., Petrasek, J., Zhang, J., Gaykova, V., Stierhof, Y. D., Dobrev, P. I., Schwarzerova, K., Rolcik, J., Seifertova, D., Luschnig, C., Benkova, E., Zazimalova, E., Geisler, M., and Friml, J. (2009). Subcellular homeostasis of phytohormone auxin is mediated by the ER-localized PIN5 transporter. Nature 459, 1136-1140.

Multani, D. S., Briggs, S. P., Chamberlin, M. A., Blakeslee, J. J., Murphy, A. S., and Johal, G. S. (2003). Loss of an MDR transporter in compact stalks of maize br2 and sorghum dw3 mutants. Science 302, 81-84.

Nardmann, J., Ji, J., Werr, W., and Scanlon, M. J. (2004). The maize duplicate genes narrow sheath1 and narrow sheath2 encode a conserved homeobox gene function in a lateral domain of shoot apical meristems. Development 131, 2827-2839.
Nardmann, J., and Werr, W. (2006) The shoot stem cell niche in angiosperms: expression patterns of WUS orthologues in rice and maize imply major modifications in the course of mono- and dicot evolution. Mol. Biol. Evol. 23, 2492-2504.

Normanly, J. (2010). Approaching cellular and molecular resolution of auxin biosynthesis and metabolism. Cold Spring Harb. Perspect. Biol. 2, a001594.

Palme, K., Dovzhenko, A., and Ditengou, F. A. (2006). Auxin transport and gravitational research: perspectives. Protoplasma 229, 175-181.

Paponov, I. A., Teale, W. D., Trebar, M. Blilou, I., and Palme, K. (2005). The PIN auxin efflux facilitators: evolutionary and functional perspectives. Trends Plant Sci. 10, 170-177.

Peer, W. A., Blakeslee, J. J., Yang, H., and Murphy, A. S. (2011). Seven things we think we know about auxin transport. Mol. Plant 4, 487-504.

Petrasek, J., and Friml, J. (2009). Auxin transport routes in plant development. Development 136, 2675-2688.

Petrasek, J., Mravec, J., Bouchard, R. Blakeslee, J. J., Abas, M., Seifertova, D., Wisniewska, J., Tadele, Z., Kubes, M., Covanova, M., Dhonukshe, P. Skupa, P., Benkova, E., Perry, L., Krecek, P., Lee, O. R., Fink, G. R., Geisler, M., Murphy, A. S., Luschnig, C., Zazimalova, E., and Friml, J. (2006). PIN proteins perform a rate-limiting function in cellular auxin efflux. Science 312, 914-918.

Pilu, R., Cassani, E., Villa, D., Curiale, S. Panzeri, D., Badone, F., and Landoni, M. (2007). Isolation and characterization of a new mutant allele of brachytic 2 maize gene. Mol. Breed. 20,83-91.

Reinhardt, D., Mandel, T., and Kuhlemeier, C. (2000). Auxin regulates the initiation and radial position of plant lateral organs. Plant Cell 12, 507-518.

Reinhardt, D., Pesce, E. R., Stieger, P., Mandel, T., Baltensperger, K., Bennett, M., Traas, J., Friml, J., and Kuhlemeier, C. (2003). Regulation of phyllotaxis by polar auxin transport. Nature 426, 255-260.

Richter, S., Anders, N., Wolters, H. Beckmann, H., Thomann, A., Heinrich, R., Schrader, J., Singh, M. K., Geldner, N., Mayer, U., and Jurgens, G. (2010). Role of the GNOM gene in Arabidopsis apicalbasal patterning-from mutant phenotype to cellular mechanism of protein action. Eur. J. Cell Biol. 89, 138-144.

Sabatini, S., Beis, D., Wolkenfelt, H., Murfett, J., Guilfoyle, T., Malamy, J.,
Benfey, P., Leyser, O., Bechtold, N., Weisbeek, P., and Scheres, B. (1999). An auxin-dependent distal organizer of pattern and polarity in the Arabidopsis root. Cell 99, 463-472.

Scarpella, E., Barkoulas, M., and Tsiantis, M. (2010). Control of leaf and vein development by auxin. Cold Spring Harb. Perspect. Biol. 2, a001511.

Scarpella, E., Marcos, D., Friml, J., and Berleth, T. (2006). Control of leaf vascular patterning by polar auxin transport. Genes Dev. 20, 1015-1027.

Schlicht, M., Strnad, M., Scanlon, M. J., Mancuso, S., Hochholdinger, F. Palme, K., Volkmann, D., Menzel, D., and Baluska, F. (2006) Auxin immunolocalization implicates vesicular neurotransmitter-like mode of polar auxin transport in root apices. Plant Signal. Behav. 1, 122-133.

Schnable, P. S., Ware, D., Fulton, R. S., Stein, J. C., Wei, F., Pasternak, S., Liang, C., Zhang, J., Fulton, L., Graves, T. A., Minx, P., Reily, A. D., Courtney, L., Kruchowski, S. S., Tomlinson, C., Strong, C., Delehaunty, K., Fronick, C., Courtney, B., Rock, S. M., Belter, E., Du, F., Kim, K., Abbott, R. M., Cotton, M., Levy, A., Marchetto, P., Ochoa, K. Jackson, S. M., Gillam, B., Chen, W., Yan, L., Higginbotham, J., Cardenas, M., Waligorski, J., Applebaum, E., Phelps, L., Falcone, J., Kanchi, K., Thane, T., Scimone, A., Thane, N., Henke, J., Wang, T., Ruppert, J., Shah, N., Rotter, K., Hodges, J., Ingenthron, E., Cordes, M., Kohlberg, S., Sgro, J., Delgado, B., Mead, K., Chinwalla, A., Leonard, S., Crouse, K., Collura, K., Kudrna, D., Currie, J., He, R., Angelova, A., Rajasekar, S., Mueller, T., Lomeli, R., Scara, G., Ko, A., Delaney, K., Wissotski, M., Lopez, G., Campos, D., Braidotti, M., Ashley, E., Golser, W., Kim, H., Lee, S., Lin, J., Dujmic, Z., Kim, W., Talag, J., Zuccolo, A., Fan, C., Sebastian, A., Kramer, M., Spiegel, L., Nascimento, L., Zutavern, T., Miller, B., Ambroise, C., Muller, S., Spooner, W., Narechania, A., Ren, L., Wei, S., Kumari, S., Faga, B., Levy, M. J., McMahan, L., Van Buren, P., Vaughn, M. W., Ying, K., Yeh, C. T., Emrich, S. J., Jia, Y., Kalyanaraman, A., Hsia, A. P., Barbazuk, W. B., Baucom, R. S., Brutnell, T. P., Carpita, N. C., Chaparro, C., Chia, J. M., Deragon, J. M., Estill, J. C., Fu, Y., Jeddeloh, J. A., Han, Y., Lee, H., Li, P., Lisch, D. R., Liu, S., Liu, Z., Nagel, D. H., McCann, M. C., SanMiguel, P., Myers, A. M., Nettleton, D., Nguyen, J., Penning, B. W., Ponnala, L., Schneider, K. L., Schwartz, 
D. C., Sharma, A., Soderlund, C., Springer, N. M., Sun, Q., Wang, H., Waterman, M., Westerman, R., Wolfgruber, T. K., Yang, L., Yu, Y., Zhang, L., Zhou, S., Zhu, Q., Bennetzen, J. L., Dawe, R. K., Jiang, J., Jiang, N., Presting, G. G., Wessler, S. R., Aluru, S., Martienssen, R. A., Clifton, S. W., McCombie, W. R., Wing, R. A., and Wilson, R. K. (2009). The B73 maize genome: complexity, diversity, and dynamics. Science 326, 1112-1115.

Sekhon, R. S., Lin, H., Childs, K. L., Hansey, C. N., Buell, C. R., de Leon, N., and Kaeppler, S. M. (2011). Genome-wide atlas of transcription during maize development. Plant J. 66, 553-563.

Shen, C., Bai, Y., Wang, S., Zhang, S., Wu, Y., Chen, M., Jiang, D., and Qi, Y. (2010). Expression profile of PIN, AUX/LAX and PGP auxin transporter gene families in Sorghum bicolor under phytohormone and abiotic stress. FEBS J. 277, 2954-2969.

Soderlund, C., Bomhoff, M., and Nelson, W. M. (2011). SyMAP v3.4: a turnkey synteny system with application to plant genomes. Nucleic Acids Res. 39, e68.

Tamura, K., Peterson, D., Peterson, N., Stecher, G., Nei, M., and Kumar, S. (2011). MEGA5: molecular evolutionary genetics analysis using maximum likelihood, evolutionary distance, and maximum parsimony methods. Mol. Biol. Evol. 28, 2731-2739.

Tanaka, H., Dhonukshe, P., Brewer, P. B., and Friml, J. (2006). Spatiotemporal asymmetric auxin distribution: a means to coordinate plant development. Cell. Mol. Life Sci. 63, 2738-2754.

Thompson, J. D., Gibson, T. J., and Higgins, D. G. (2002). Multiple sequence alignment using ClustalW and ClustalX. Curr. Protoc. Bioinformatics 2, 2.3.
Titapiwatanakun, B., and Murphy, A. S. (2008). Post-transcriptional regulation of auxin transport proteins: cellular trafficking, protein phosphorylation, protein maturation, ubiquitination, and membrane composition. J. Exp. Bot. 60, 1093-1107.

Tuskan, G. A., Difazio, S., Jansson, S. Bohlmann, J., Grigoriev, I., Hellsten, U., Putnam, N., Ralph, S., Rombauts, S., Salamov, A., Schein, J., Sterck, L., Aerts, A., Bhalerao, R. R., Bhalerao, R. P., Blaudez, D., Boerjan, W., Brun, A., Brunner, A., Busov, V., Campbell, M., Carlson, J., Chalot, M., Chapman, J., Chen, G. L., Cooper, D., Coutinho, P. M., Couturier, J., Covert, S., Cronk, Q., Cunningham, R., Davis, J., Degroeve, S., Dejardin, A., Depamphilis, C., Detter, J., Dirks, B., Dubchak, I., Duplessis, S., Ehlting, J., Ellis, B., Gendler, K., Goodstein, D., Gribskov, M., Grimwood, J., Groover, A., Gunter, L., Hamberger, B., Heinze, B., Helariutta, Y., Henrissat, B., Holligan, D., Holt, R., Huang, W., Islam-Faridi, N., Jones, S., Jones-Rhoades, M., Jorgensen, R., Joshi, C., Kangasjarvi, J., Karlsson, J., Kelleher, C., Kirkpatrick, R., Kirst, M., Kohler, A., Kalluri, U., Larimer, F., Leebens-Mack, J., Leple, J. C., Locascio, P., Lou, Y., Lucas, S., Martin, F., Montanini, B., Napoli, C., Nelson, D. R., Nelson, C., Nieminen, K., Nilsson, O., Pereda, V., Peter, G., Philippe, R., Pilate, G., Poliakov, A., Razumovskaya, J., Richardson, P., Rinaldi, C., Ritland, K., Rouze, P., Ryaboy, D., Schmutz, J., Schrader, J., Segerman, B., Shin, H., Siddiqui, A., Sterky, F., Terry, A., Tsai, C. J., Uberbacher, E., Unneberg, P., Vahala, J., Wall, K., Wessler, S., Yang, G., Yin, T., Douglas, C., Marra, M., Sandberg, G., Van de Peer, Y., and Rokhsar, D. (2006). The genome of black cottonwood, Populus trichocarpa (Torr. \& Gray). Science 313, 1596-1604.
Varotto, S., Locatelli, S., Canova, S. Pipal, A., Motto, M., and Rossi, V. (2003). Expression profile and cellular localization of maize Rpd3-type histone deacetylases during plant development. Plant Physiol. 133, 606-617.

Verrier, P. J., Bird, D., Burla, B., Dassa, E. Forestier, C., Geisler, M., Klein, M., Kolukisaoglu, U., Lee, Y., Martinoia, E., Murphy, A., Rea, P. A., Samuels, L. Schulz, B., Spalding, E. J., Yazaki, K., and Theodoulou, F. L. (2008). Plant $\mathrm{ABC}$ proteins - a unified nomenclature and updated inventory. Trends Plant Sci. 13, 151-159.

Vieten, A., Sauer, M., Brewer, P. B. and Friml, J. (2007). Molecular and cellular aspects of auxin-transportmediated development. Trends Plant Sci. 12, 160-168.

Wabnik, K., Kleine-Vehn, J., Govaerts, W., and Friml, J. (2011). Prototype cell-to-cell auxin transport mechanism by intracellular auxin compartmentalization. Trends Plant Sci. 16 468-475.

Wang, J., Hu, H., Wang, G., Li, J., Chen, J., and Wu, P. (2009). Expression of PIN genes in rice (Oryza sativa L.): tissue specificity and regulation by hormones. Mol. Plant. 2, 823-831.

Weijers, D., Sauer, M., Meurette, O. Friml, J., Ljung, K., Sandberg, G., Hooykaas, P., and Offringa, R. (2005). Maintenance of embryonic auxin distribution for apicalbasal patterning by PIN-FORMEDdependent auxin transport in Arabidopsis. Plant Cell 17, 2517-2526.

Woll, K., Borsuk, L. A., Stransky, H., Nettleton, D., Schnable, P. S., and Hochholdinger, F. (2005). Isolation, characterization, and pericyclespecific transcriptome analyses of the novel maize lateral and seminal root initiation mutant rum1. Plant Physiol. 139, 1255-1267.

$\mathrm{Xu}$, M., Zhu, L., Shou, H., and Wu, P. (2005). A PIN1 family gene, OsPIN1, involved in auxin-dependent adventitious root emergence and tillering in rice. Plant Cell Physiol. 46, 1674-1681.

Yang, H., and Murphy, A. S. (2009). Functional expression and characterization of Arabidopsis ABCB, AUX 1 and PIN auxin transporters in Schizosaccharomyces pombe. Plant J. 59, 179-191.

Zazimalova, E., Krecek, P., Skupa, P., Hoyerova, K., and Petrasek, J. (2007). Polar transport of the plant hormone auxin - the role of PINFORMED (PIN) proteins. Cell. Mol. Life Sci. 64, 1621-1637.

Zazimalova, E., Murphy, A. S., Yang, H., Hoyerova, K., and Hosek, P. (2010). Auxin transporters - why so many? Cold Spring Harb. Perspect. Biol. 2, a001552.

Zhao, Y. (2010). Auxin biosynthesis and its role in plant development. Annu. Rev. Plant Biol. 61, 49-64.

Conflict of Interest Statement: The authors declare that the research was conducted in the absence of any commercial or financial relationships that could be construed as a potential conflict of interest.

Received: 14 October 2011; accepted: 17 January 2012; published online: 08 February 2012.

Citation: Forestan C, Farinati $S$ and Varotto $S$ (2012) The maize PIN gene family of auxin transporters. Front. Plant Sci. 3:16. doi: 10.3389/fpls.2012.00016 This article was submitted to Frontiers in Plant Traffic and Transport, a specialty of Frontiers in Plant Science.

Copyright (C) 2012 Forestan, Farinati and Varotto. This is an open-access article distributed under the terms of the Creative Commons Attribution Non Commercial License, which permits noncommercial use, distribution, and reproduction in other forums, provided the original authors and source are credited. 